\title{
AN AUGMENTED MIXED FINITE ELEMENT METHOD FOR THE NAVIER-STOKES EQUATIONS WITH VARIABLE VISCOSITY*
}

\author{
JESSIKA CAMAÑO ${ }^{\dagger}$, GABRIEL N. GATICA $^{\ddagger}$, RICARDO OYARZÚA $^{\S}$, AND \\ GIORDANO TIERRA
}

\begin{abstract}
A new mixed variational formulation for the Navier-Stokes equations with constant density and variable viscosity depending nonlinearly on the gradient of velocity, is proposed and analyzed here. Our approach employs a technique previously applied to the stationary Boussinesq problem and to the Navier-Stokes equations with constant viscosity, which consists firstly of the introduction of a modified pseudostress tensor involving the diffusive and convective terms, and the pressure. Next, by using an equivalent statement suggested by the incompressibility condition, the pressure is eliminated, and in order to handle the nonlinear viscosity, the gradient of velocity is incorporated as an auxiliary unknown. Furthermore, since the convective term forces the velocity to live in a smaller space than usual, we overcome this difficulty by augmenting the variational formulation with suitable Galerkin-type terms arising from the constitutive and equilibrium equations, the aforementioned relation defining the additional unknown, and the Dirichlet boundary condition. The resulting augmented scheme is then written equivalently as a fixed point equation, and hence the well-known Schauder and Banach theorems, combined with classical results on bijective monotone operators, are applied to prove the unique solvability of the continuous and discrete systems. No discrete inf-sup conditions are required for the well-posedness of the Galerkin scheme, and hence arbitrary finite element subspaces of the respective continuous spaces can be utilized. In particular, given an integer $k \geq 0$, piecewise polynomials of degree $\leq k$ for the gradient of velocity, Raviart-Thomas spaces of order $k$ for the pseudostress, and continuous piecewise polynomials of degree $\leq k+1$ for the velocity, constitute feasible choices. Finally, optimal a priori error estimates are derived, and several numerical results illustrating the good performance of the augmented mixed finite element method and confirming the theoretical rates of convergence are reported.
\end{abstract}

Key words. Navier-Stokes equations, nonlinear viscosity, augmented mixed formulation, fixed point theory, mixed finite element methods, a priori error analysis

AMS subject classifications. 65N30, 65N12, 65N15, 35Q79, 80A20, 76R05, 76D07

\section{DOI. $10.1137 / 15 \mathrm{M} 1013146$}

1. Introduction. The utilization of pseudostress-based approaches for Newtonian and non-Newtonian incompressible flows has gained considerable attention in recent years due to their natural way of circumventing the usual symmetry requirement

*Received by the editors March 19, 2015; accepted for publication (in revised form) January 28, 2016; published electronically April 12, 2016. The research of the authors was partially supported by CONICYT-Chile through BASAL project CMM, Universidad de Chile, project Anillo ACT1118 (ANANUM), project Inserción de Capital Humano Avanzado en la Academia 79130048, and projects Fondecyt 11121347 and 11140691; by Centro de Investigación en Ingeniería Matemática (CI ${ }^{2} \mathrm{MA}$ ), Universidad de Concepción; by Universidad del Bío-Bío through DIUBB project 120808 GI/EF; and by Ministry of Education, Youth and Sports of the Czech Republic through the ERC-CZ project LL1202.

http://www.siam.org/journals/sinum/54-2/M101314.html

†Departamento de Matemática y Física Aplicadas, Universidad Católica de la Santísima Concepción, Casilla 297, Concepción, Chile, and CI ${ }^{2} \mathrm{MA}$, Universidad de Concepción, Casilla 160-C, Concepción, Chile (jecamano@ucsc.cl).

${ }^{\ddagger} \mathrm{CI}^{2} \mathrm{MA}$ and Departamento de Ingeniería Matemática, Universidad de Concepción, Casilla 160-C, Concepción, Chile (ggatica@ci2ma.udec.cl).

$\S$ GIMNAP-Departamento de Matemática, Universidad del Bío-Bío, Casilla 5-C, Concepción, Chile, and $\mathrm{CI}^{2} \mathrm{MA}$, Universidad de Concepción, Casilla 160-C, Concepción, Chile (royarzua@ubiobio. $\mathrm{cl})$.

IMathematical Institute, Faculty of Mathematics and Physics, Charles University, Prague 8, 186 75, Czech Republic. Current address: Department of Mathematics, Temple University, Philadelphia, PA, 19122 (gtierra@temple.edu). 
of the stress-based formulations. In this direction, and especially in the context of least-squares and augmented methods, two new procedures have arisen: the velocitypressure-pseudostress and velocity-pseudostress formulations (see, e.g., [7], [8], [24]). In particular, augmented mixed finite element methods for both pseudostress-based formulations of the stationary Stokes equations, which extend analogue results for linear elasticity problems (see [25], [26], [30]), were introduced and analyzed in [24]. In turn, the pure velocity-pseudostress formulation of the Stokes equations, that is without augmenting or employing least-squares terms, was first studied in [9], whereas the utilization of the stress variable, yielding the computation of the symmetric part of the velocity gradient only, was considered in [36]. The corresponding augmented mixed finite element scheme for the stress-based formulation of the Stokes problem, in which the vorticity is introduced as the Lagrange multiplier taking care of the weak symmetry of the stress, was studied in [23]. Now, going back to the pseudostress formulations, we remark that the approach from [9] was reconsidered in [32] where further results, including the eventual incorporation of the pressure unknown and a posteriori error analysis, were provided. Furthermore, the velocity-pressurepseudostress formulation has also been applied to nonlinear Stokes problems. In particular, a new mixed finite element method for a class of models arising in quasiNewtonian fluids, was introduced in [28]. The results in [28] were extended in [17] to a setting in reflexive Banach spaces, thus allowing other nonlinear models such as the Carreau law for viscoplastic flows. Moreover, the dual-mixed approach from [28] and [17] was reformulated in [35] by restricting the space for the velocity gradient to that of trace-free tensors. As a consequence, the pressure is eliminated and a three-field formulation with the pseudostress, the velocity, and the velocity gradient as unknowns, is obtained. In addition, the approach from [32] was extended in [33] to the class of nonlinear problems originally studied in [28] and [35]. For other contributions dealing with stress- or pseudostress-based formulations in incompressible flows, including nonlinear and transmission problems, and corresponding a posteriori error analyses, we refer to [18], [19], [22], [31], and the references therein.

On the other hand, in connection with the utilization of diverse dual-mixed approaches for the Navier-Stokes equations, we can mention [10], [11], [12], [20], [21], and [37]. In particular, the velocity-pseudostress approach from [9], which employs the usual pseudostress tensor depending linearly on the gradient of velocity and the pressure, is extended in [10] to the aforementioned nonlinear problem. The well-posedness of the continuous formulation is established through its equivalence with the classical velocity-pressure setting, whereas the discrete scheme is analyzed by employing the theory from [6] for the approximation of branches of nonsingular solutions. The corresponding velocity-pressure-pseudostress formulation for the Navier-Stokes equations was developed later on in [12]. In turn, a dual-mixed formulation of the Navier-Stokes system with Dirichlet boundary conditions, in which the gradient of the velocity is introduced as a new unknown, is proposed and analyzed in [21]. Quasi-optimal a priori error estimates and an associated a posteriori error analysis are derived there. More recently, a new dual-mixed method, in which the main unknowns are given by the velocity, its gradient, and a modified nonlinear pseudostress tensor linking the usual stress and the convective term, has been proposed in [37]. The Babuška-Brezzi theory and a fixed point argument are employed there to prove the well-posedness of the continuous formulation. However, in order to satisfy the inf-sup conditions required by the discrete analysis, new but more expensive finite element subspaces than usual had to be introduced in [37]. Lately, the idea from [37] has been modified in [13] through 
the introduction of a nonlinear pseudostress tensor linking now the pseudostress (instead of the stress) and the convective term, which, together with the velocity, constitute the only unknowns. The resulting mixed formulation is then suitably augmented with Galerkin-type terms arising from the constitutive and equilibrium equations, and the Dirichlet boundary condition, so that the Banach fixed point and Lax-Milgram theorems are applied to conclude the well-posedness of the continuous and discrete formulations. The approach from [13] has been further extended in [16], where a new augmented mixed-primal formulation for the stationary Boussinesq problem (cf. [39]) has been proposed and analyzed. In addition to the methods and tools employed in [13], the analysis in [16] makes use of the Babuška-Brezzi theory and the Brouwer fixed point theorem. Finally, within a slightly different perspective, we can also refer to [14], where stabilized three-field (deviatoric stress, velocity, and pressure) finite element formulations of the Navier-Stokes problem for quasi-Newtonian fluids, are proposed and analyzed. Two stabilized schemes of sub-grid-scale-type are introduced there, which allow the use of the same polynomial degree for the three unknowns, even in cases where the convection component is dominant and the velocity gradients are high.

According to the above bibliographic discussion, the purpose of the present paper is to additionally contribute in the direction suggested by [37], [13], and [16], by extending the analysis and results from [13] to the Navier-Stokes equations with constant density and variable viscosity. Indeed, the viscosity of many fluids, including on one hand biological ones such as blood, and on the other hand polymers and molten metals, among others, may depend on the state variables. In particular, here we are interested in developing a mixed finite element approach for those quasi-Newtonian fluids whose viscosity is a nonlinear function of the magnitude of the gradient of velocity. For this purpose, we first introduce the same modified pseudostress tensor from [13], and then, utilizing an equivalent statement arising from the incompressibility condition, eliminate the pressure unknown. In addition, similarly to [37] and [31], the gradient of velocity is incorporated as an auxiliary unknown, which allows us to handle the nonlinear viscosity within the dual-mixed setting. In turn, the eventual difficulty arising from the fact that the velocity actually lives in a smaller space, is overcomed by adopting the same procedure from [13] (see also [33] and several previous references therein), that is by incorporating suitable Galerkintype terms into the formulation. As a further consequence, and differently from [37], no discrete inf-sup conditions are required for the well-posedness of the associated Galerkin scheme. The rest of the paper is organized as follows. In section 2 we first describe some standard notations and functional spaces, and then introduce the model problem of interest and set the definite unknowns to be considered in the variational formulation. Next, in section 3 we derive the augmented mixed variational formulation, introduce and analyze the equivalent fixed point setting, and conclude the corresponding well-posedness result assuming sufficiently small data. The associated Galerkin scheme is then studied in section 4 by employing a discrete version of the fixed point strategy developed in section 3. We emphasize that no discrete inf-sup conditions are required for the discrete analysis, and therefore arbitrary finite element subspaces can be employed. In addition, under a similar assumption on the size of the data, the corresponding a priori error estimate is also deduced there by applying a suitable Strang-type lemma for nonlinear problems. Finally, in section 5 we present several numerical examples illustrating the good performance of the augmented mixed finite element method and confirming the theoretical rates of convergence. 


\section{The model problem.}

2.1. Preliminaries. Let us denote by $\Omega \subseteq \mathrm{R}^{n}, n \in\{2,3\}$, a given bounded domain with polyhedral boundary $\Gamma$, and denote by $\boldsymbol{\nu}$ the outward unit normal vector on $\Gamma$. Standard notation will be adopted for Lebesgue spaces $\mathrm{L}^{p}(\Omega)$ and Sobolev spaces $\mathrm{H}^{s}(\Omega)$ with norm $\|\cdot\|_{s, \Omega}$ and seminorm $|\cdot|_{s, \Omega}$. In particular, $\mathrm{H}^{1 / 2}(\Gamma)$ is the space of traces of functions of $\mathrm{H}^{1}(\Omega)$ and $\mathrm{H}^{-1 / 2}(\Gamma)$ denotes its dual. By $\mathbf{M}$ and $\mathbb{M}$ we will denote the corresponding vectorial and tensorial counterparts of the generic scalar functional space $\mathrm{M}$, and $\|\cdot\|$, with no subscripts, will stand for the natural norm of either an element or an operator in any product functional space. In turn, for any vector fields $\boldsymbol{v}=\left(v_{i}\right)_{i=1, n}$ and $\boldsymbol{w}=\left(w_{i}\right)_{i=1, n}$, we set the gradient, divergence, and tensor product operators, as

$$
\nabla \boldsymbol{v}:=\left(\frac{\partial v_{i}}{\partial x_{j}}\right)_{i, j=1, n}, \quad \operatorname{div} \boldsymbol{v}:=\sum_{j=1}^{n} \frac{\partial v_{j}}{\partial x_{j}}, \quad \text { and } \quad \boldsymbol{v} \otimes \boldsymbol{w}:=\left(v_{i} w_{j}\right)_{i, j=1, n}
$$

In addition, for any tensor fields $\boldsymbol{\tau}=\left(\tau_{i j}\right)_{i, j=1, n}$ and $\boldsymbol{\zeta}=\left(\zeta_{i j}\right)_{i, j=1, n}$, we let $\operatorname{div} \boldsymbol{\tau}$ be the divergence operator div acting along the rows of $\boldsymbol{\tau}$, and define the transpose, the trace, the tensor inner product, and the deviatoric tensor, respectively, as

$$
\boldsymbol{\tau}^{\mathrm{t}}:=\left(\tau_{j i}\right)_{i, j=1, n}, \quad \operatorname{tr}(\boldsymbol{\tau}):=\sum_{i=1}^{n} \tau_{i i}, \quad \boldsymbol{\tau}: \boldsymbol{\zeta}:=\sum_{i, j=1}^{n} \tau_{i j} \zeta_{i j}, \quad \text { and } \quad \boldsymbol{\tau}^{\mathrm{d}}:=\boldsymbol{\tau}-\frac{1}{n} \operatorname{tr}(\boldsymbol{\tau}) \mathbb{I} .
$$

Furthermore, we recall that

$$
\mathbb{H}(\operatorname{div} ; \Omega):=\left\{\boldsymbol{\tau} \in \mathbb{L}^{2}(\Omega): \quad \operatorname{div} \boldsymbol{\tau} \in \mathbf{L}^{2}(\Omega)\right\},
$$

equipped with the usual norm

$$
\|\boldsymbol{\tau}\|_{\operatorname{div} ; \Omega}^{2}:=\|\boldsymbol{\tau}\|_{0, \Omega}^{2}+\|\operatorname{div} \boldsymbol{\tau}\|_{0, \Omega}^{2},
$$

is a standard Hilbert space in the realm of mixed problems. Finally, in what follows $\mathbb{I}$ stands for the identity tensor in $\mathbb{R}:=\mathrm{R}^{n \times n}$, and $|\cdot|$ denotes the Euclidean norm in $\mathbf{R}:=\mathrm{R}^{n}$.

2.2. The Navier-Stokes equations with variable viscosity. We consider the Navier-Stokes equations with constant density and variable viscosity, that is

$$
\begin{aligned}
& -\operatorname{div}(\mu(|\nabla \boldsymbol{u}|) \nabla \boldsymbol{u})+(\nabla \boldsymbol{u}) \boldsymbol{u}+\nabla p=\boldsymbol{f} \quad \text { in } \quad \Omega, \\
& \operatorname{div} \boldsymbol{u}=0 \quad \text { in } \Omega, \\
& \boldsymbol{u}=\boldsymbol{g} \quad \text { on } \quad \Gamma,
\end{aligned}
$$

where the unknowns are the velocity $\boldsymbol{u}$ and the pressure $p$ of a fluid occupying the region $\Omega$. The given data are a function $\mu: \mathrm{R}^{+} \longrightarrow \mathrm{R}$ describing the nonlinear viscosity, a volume force $\boldsymbol{f} \in \mathbf{L}^{2}(\Omega)$, and the boundary velocity $\boldsymbol{g} \in \mathbf{H}^{1 / 2}(\Gamma)$. Note that $\boldsymbol{g}$ must satisfy the compatibility condition

$$
\int_{\Gamma} \boldsymbol{g} \cdot \boldsymbol{\nu}=0
$$

Copyright $@$ by SIAM. Unauthorized reproduction of this article is prohibited. 
which comes from the incompressibility condition of the fluid, and that uniqueness of a pressure solution of $(2.1)$ is ensured in the space

$$
\mathrm{L}_{0}^{2}(\Omega)=\left\{q \in L^{2}(\Omega): \quad \int_{\Omega} q=0\right\} .
$$

Furthermore, we assume that $\mu$ is of class $C^{1}$, and that there exist constants $\mu_{1}, \mu_{2}>$ 0 , such that

$$
\mu_{1} \leq \mu(s) \leq \mu_{2} \quad \text { and } \quad \mu_{1} \leq \mu(s)+s \mu^{\prime}(s) \leq \mu_{2} \quad \forall s \geq 0,
$$

which, according to the result provided by [34, Theorem 3.8], imply Lipschitz continuity and strong monotonicity of the nonlinear operator induced by $\mu$. We will go back to this fact later on in section 3. In addition, it is easy to see that the forthcoming analysis also applies to the slightly more general case of a viscosity function acting on $\Omega \times \mathrm{R}^{+}$, that is $\mu: \Omega \times \mathrm{R}^{+} \longrightarrow \mathrm{R}$. Some examples of nonlinear $\mu$ are the following:

$$
\mu(s):=2+\frac{1}{1+s} \text { and } \mu(s):=\alpha_{0}+\alpha_{1}\left(1+s^{2}\right)^{(\beta-2) / 2},
$$

where $\alpha_{0}, \alpha_{1}>0$ and $\beta \in[1,2]$. The first example is basically academic but the second one corresponds to a particular case of the well-known Carreau law in fluid mechanics. It is easy to see that they both satisfy $(2.3)$ with $\left(\mu_{1}, \mu_{2}\right)=(2,3)$ and $\left(\mu_{1}, \mu_{2}\right)=\left(\alpha_{0}, \alpha_{0}+\alpha_{1}\right)$, respectively.

Next, following [13] and [16], we observe that the first equation in (2.1) can be rewritten as the equilibrium equation

$$
-\operatorname{div} \sigma=f \quad \text { in } \Omega,
$$

where $\boldsymbol{\sigma}$ is the tensor unknown defined by

$$
\boldsymbol{\sigma}:=\mu(|\nabla \boldsymbol{u}|) \nabla \boldsymbol{u}-(\boldsymbol{u} \otimes \boldsymbol{u})-p \mathbb{I} \quad \text { in } \quad \Omega .
$$

Moreover, it is straightforward to see that (2.6) together with the incompressibility condition are equivalent to the pair of equations given by

$$
\begin{array}{r}
\mu(|\nabla \boldsymbol{u}|) \nabla \boldsymbol{u}-(\boldsymbol{u} \otimes \boldsymbol{u})^{\mathrm{d}}=\boldsymbol{\sigma}^{\mathrm{d}} \quad \text { in } \quad \Omega, \\
p=-\frac{1}{n} \operatorname{tr}(\boldsymbol{\sigma}+\boldsymbol{u} \otimes \boldsymbol{u}) \quad \text { in } \quad \Omega .
\end{array}
$$

In this way, eliminating the pressure unknown (which, anyway, can be approximated later on by the postprocessed formula suggested by the second equation of (2.7)), we arrive, at first instance, at the following system of equations with unknowns $\boldsymbol{u}$ and $\boldsymbol{\sigma}$,

$$
\begin{aligned}
\mu(|\nabla \boldsymbol{u}|) \nabla \boldsymbol{u}-(\boldsymbol{u} \otimes \boldsymbol{u})^{\mathrm{d}} & =\boldsymbol{\sigma}^{\mathrm{d}} & & \text { in } \Omega, \\
-\operatorname{div} \boldsymbol{\sigma} & =\boldsymbol{f} & & \text { in } \Omega, \\
\boldsymbol{u} & =\boldsymbol{g} & & \text { on } \quad \Gamma, \\
\int_{\Omega} \operatorname{tr}(\boldsymbol{\sigma}+\boldsymbol{u} \otimes \boldsymbol{u}) & =0 . & &
\end{aligned}
$$

We remark here that the incompressibility of the fluid is implicitly present in the new constitutive equation relating $\boldsymbol{\sigma}$ and $\boldsymbol{u}$ (first equation of (2.8)). In turn, the fact that the pressure $p$ must belong to $\mathrm{L}_{0}^{2}(\Omega)$ (as said before) is guaranteed by the equivalent statement given by the last equation of $(2.8)$.

Copyright $@$ by SIAM. Unauthorized reproduction of this article is prohibited. 
Finally, since we are interested in a mixed variational formulation of our nonlinear problem, and in order to employ the integration by parts formula that is usually required by this approach, we also introduce the auxiliary unknown $\boldsymbol{t}:=\nabla \boldsymbol{u}$ in $\Omega$. Consequently, instead of (2.8), we consider from now on the set of equations with unknowns $\boldsymbol{t}, \boldsymbol{u}$, and $\boldsymbol{\sigma}$, given by

$$
\begin{aligned}
\nabla \boldsymbol{u} & =\boldsymbol{t} & & \text { in } \Omega, \\
\mu(|\boldsymbol{t}|) \boldsymbol{t}-(\boldsymbol{u} \otimes \boldsymbol{u})^{\mathrm{d}} & =\boldsymbol{\sigma}^{\mathrm{d}} & & \text { in } \Omega, \\
-\operatorname{div} \boldsymbol{\sigma} & =\boldsymbol{f} & & \text { in } \Omega, \\
\boldsymbol{u} & =\boldsymbol{g} & & \text { on } \quad \Gamma, \\
\int_{\Omega} \operatorname{tr}(\boldsymbol{\sigma}+\boldsymbol{u} \otimes \boldsymbol{u}) & =0 . & &
\end{aligned}
$$

\section{The continuous formulation.}

3.1. The augmented mixed formulation. We now proceed to derive a weak formulation of (2.9). We begin by recalling (see, e.g., [5], [27]) that there holds

$$
\mathbb{H}(\operatorname{div} ; \Omega)=\mathbb{H}_{0}(\operatorname{div} ; \Omega) \oplus \mathrm{R} \mathbb{I},
$$

where

$$
\mathbb{H}_{0}(\operatorname{div} ; \Omega):=\left\{\boldsymbol{\zeta} \in \mathbb{H}(\operatorname{div} ; \Omega): \quad \int_{\Omega} \operatorname{tr}(\boldsymbol{\zeta})=0\right\} .
$$

Equivalently, each $\boldsymbol{\zeta} \in \mathbb{H}(\mathbf{d i v} ; \Omega)$ can be decomposed as $\boldsymbol{\zeta}=\boldsymbol{\zeta}_{0}+d \mathbb{I}$, with

$$
\boldsymbol{\zeta}_{0}:=\boldsymbol{\zeta}-\left(\frac{1}{n|\Omega|} \int_{\Omega} \operatorname{tr}(\boldsymbol{\zeta})\right) \mathbb{I} \in \mathbb{H}_{0}(\operatorname{div} ; \Omega) \quad \text { and } \quad d:=\frac{1}{n|\Omega|} \int_{\Omega} \operatorname{tr}(\boldsymbol{\zeta}) \in \mathrm{R} .
$$

In particular, decomposing $\boldsymbol{\sigma}$ in (2.9) as $\boldsymbol{\sigma}=\boldsymbol{\sigma}_{0}+c \mathbb{I}$, with $\boldsymbol{\sigma}_{0} \in \mathbb{H}_{0}(\boldsymbol{d i v} ; \Omega)$, we deduce from (3.2) and the last equation in (2.9) that $c$ is given explicity in terms of $\boldsymbol{u}$ as

$$
c=-\frac{1}{n|\Omega|} \int_{\Omega} \operatorname{tr}(\boldsymbol{u} \otimes \boldsymbol{u}) .
$$

In this way, since $\boldsymbol{\sigma}^{\mathrm{d}}=\boldsymbol{\sigma}_{0}^{\mathrm{d}}$ and $\operatorname{div} \boldsymbol{\sigma}=\operatorname{div} \boldsymbol{\sigma}_{0}$, throughout the rest of the paper we rename $\boldsymbol{\sigma}_{0}$ as $\boldsymbol{\sigma} \in \mathbb{H}_{0}(\operatorname{div} ; \Omega)$ and realize that the second and third equations of (2.9) remain unchanged. In addition, thanks to the incompressibility condition and the first equation of (2.9), we can look for the unknown $\boldsymbol{t}$ in the space

$$
\mathbb{L}_{\mathrm{tr}}^{2}(\Omega):=\left\{s \in \mathbb{L}^{2}(\Omega): \quad \operatorname{tr} s=0\right\} .
$$

Thus, multiplying the first equation of (2.9) by a test function $\boldsymbol{\tau} \in \mathbb{H}(\operatorname{div} ; \Omega)$, noting under the above constraint for $\boldsymbol{t}$ that $\int_{\Omega} \boldsymbol{\tau}: \boldsymbol{t}=\int_{\Omega} \tau^{\mathrm{d}}: \boldsymbol{t}$, and using the Dirichlet condition for $\boldsymbol{u}$, we get

$$
\int_{\Omega} \tau^{\mathrm{d}}: \boldsymbol{t}+\int_{\Omega} \boldsymbol{u} \cdot \operatorname{div} \boldsymbol{\tau}=\langle\boldsymbol{\tau} \boldsymbol{\nu}, \boldsymbol{g}\rangle \quad \forall \boldsymbol{\tau} \in \mathbb{H}(\operatorname{div} ; \Omega),
$$

where $\langle\cdot, \cdot\rangle$ stands for the duality pairing between $\mathbf{H}^{-1 / 2}(\Gamma)$ and $\mathbf{H}^{1 / 2}(\Gamma)$. Moreover, it is easy to see that (3.4) is actually satisfied in advance for $\boldsymbol{\tau}=d \mathbb{I}$ with $d \in \mathrm{R}$, since in this case all the terms appearing there vanish. In particular, the compatibility condition (2.2) explains this fact for the boundary term. According to this and the 
decomposition (3.1), we deduce that (3.4) can be stated, equivalently, as

$$
\int_{\Omega} \tau^{\mathrm{d}}: \boldsymbol{t}+\int_{\Omega} \boldsymbol{u} \cdot \operatorname{div} \boldsymbol{\tau}=\langle\boldsymbol{\tau} \boldsymbol{\nu}, \boldsymbol{g}\rangle \quad \forall \boldsymbol{\tau} \in \mathbb{H}_{0}(\operatorname{div} ; \Omega) .
$$

Similarly, since the traces of $\boldsymbol{t},(\boldsymbol{u} \otimes \boldsymbol{u})^{\mathrm{d}}$, and $\boldsymbol{\sigma}^{\mathrm{d}}$ all vanish, and since there also holds the decomposition $\mathbb{L}^{2}(\Omega)=\mathbb{L}_{\mathrm{tr}}^{2}(\Omega) \oplus \mathrm{R} \mathbb{I}$, we realize that the constitutive equation given by the second equation of (2.9) needs to be tested only against $s \in \mathbb{L}_{\mathrm{tr}}^{2}(\Omega)$, which yields

$$
\int_{\Omega} \mu(|\boldsymbol{t}|) \boldsymbol{t}: s-\int_{\Omega} \boldsymbol{\sigma}^{\mathrm{d}}: s-\int_{\Omega}(\boldsymbol{u} \otimes \boldsymbol{u})^{\mathrm{d}}: s=0 \quad \forall s \in \mathbb{L}_{\mathrm{tr}}^{2}(\Omega) .
$$

In turn, the equilibrium equation given by the third equation of (2.9) is rewritten as

$$
-\int_{\Omega} \boldsymbol{v} \cdot \operatorname{div} \boldsymbol{\sigma}=\int_{\Omega} \boldsymbol{f} \cdot \boldsymbol{v} \quad \forall \boldsymbol{v} \in \mathbf{L}^{2}(\Omega) .
$$

We have thus arrived, at first instance, at the following weak formulation of (2.9): Find $(\boldsymbol{t}, \boldsymbol{\sigma}) \in \mathbb{L}_{\mathrm{tr}}^{2}(\Omega) \times \mathbb{H}_{0}(\operatorname{div} ; \Omega)$, and $\boldsymbol{u}$ in a suitable space, such that

$$
\begin{aligned}
\int_{\Omega} \mu(|\boldsymbol{t}|) \boldsymbol{t}: \boldsymbol{s}-\int_{\Omega} \boldsymbol{\sigma}^{\mathrm{d}}: \boldsymbol{s}-\int_{\Omega}(\boldsymbol{u} \otimes \boldsymbol{u})^{\mathrm{d}}: \boldsymbol{s} & =0 & & \forall s \in \mathbb{L}_{\text {tr }}^{2}(\Omega), \\
\int_{\Omega} \boldsymbol{\tau}^{\mathrm{d}}: \boldsymbol{t}+\int_{\Omega} \boldsymbol{u} \cdot \operatorname{div} \boldsymbol{\tau} & =\langle\boldsymbol{\tau} \boldsymbol{\nu}, \boldsymbol{g}\rangle & & \forall \boldsymbol{\tau} \in \mathbb{H}_{0}(\operatorname{div} ; \Omega), \\
-\int_{\Omega} \boldsymbol{v} \cdot \operatorname{div} \boldsymbol{\sigma} & =\int_{\Omega} \boldsymbol{f} \cdot \boldsymbol{v} & & \forall \boldsymbol{v} \in \mathbf{L}^{2}(\Omega) .
\end{aligned}
$$

We continue our analysis by observing that the third term in the first row of the foregoing system requires $\boldsymbol{u}$ to live in a smaller space than $\mathbf{L}^{2}(\Omega)$. In fact, by applying Cauchy-Schwarz and Hölder inequalities, and then employing the compact (and hence continuous) injection $\boldsymbol{i}_{c}$ of $\mathbf{H}^{1}(\Omega)$ into $\mathbf{L}^{4}(\Omega)$ (see the Rellich-Kondrachov compactness theorem in [1, Theorem 6.3] or [40, Theorem 1.3.5]), and denoting $c_{1}(\Omega):=\left\|\boldsymbol{i}_{c}\right\|^{2}$, we find that there holds

$$
\left|\int_{\Omega}(\boldsymbol{u} \otimes \boldsymbol{w})^{\mathrm{d}}: \boldsymbol{s}\right| \leq\|\boldsymbol{u}\|_{\mathbf{L}^{4}(\Omega)}\|\boldsymbol{w}\|_{\mathbf{L}^{4}(\Omega)}\|\boldsymbol{s}\|_{0, \Omega} \leq c_{1}(\Omega)\|\boldsymbol{u}\|_{1, \Omega}\|\boldsymbol{w}\|_{1, \Omega}\|\boldsymbol{s}\|_{0, \Omega}
$$

for all $\boldsymbol{u}, \boldsymbol{w} \in \mathbf{H}^{1}(\Omega), \boldsymbol{s} \in \mathbf{L}^{2}(\Omega)$, which suggests to look for the unknown $\boldsymbol{u}$ in $\mathbf{H}^{1}(\Omega)$ and to restrict the set of corresponding test functions $\boldsymbol{v}$ to the same space. Consequently, and in order to be able to analyze the present variational formulation of (2.9), we now augment (3.5) through the incorporation of the following redundant Galerkin terms:

$$
\begin{aligned}
\kappa_{1} \int_{\Omega}\left\{\boldsymbol{\sigma}^{\mathrm{d}}-\mu(|\boldsymbol{t}|) \boldsymbol{t}+(\boldsymbol{u} \otimes \boldsymbol{u})^{\mathrm{d}}\right\}: \boldsymbol{\tau}^{\mathrm{d}}=0 & \forall \boldsymbol{\tau} \in \mathbb{H}_{0}(\operatorname{div} ; \Omega), \\
\kappa_{2} \int_{\Omega} \operatorname{div} \boldsymbol{\sigma} \cdot \operatorname{div} \boldsymbol{\tau}=-\kappa_{2} \int_{\Omega} \boldsymbol{f} \cdot \operatorname{div} \boldsymbol{\tau} & \forall \boldsymbol{\tau} \in \mathbb{H}_{0}(\operatorname{div} ; \Omega), \\
\kappa_{3} \int_{\Omega}\{\nabla \boldsymbol{u}-\boldsymbol{t}\}: \nabla \boldsymbol{v}=0 & \forall \boldsymbol{v} \in \mathbf{H}^{1}(\Omega), \\
\kappa_{4} \int_{\Gamma} \boldsymbol{u} \cdot \boldsymbol{v}=\kappa_{4} \int_{\Gamma} \boldsymbol{g} \cdot \boldsymbol{v} & \forall \boldsymbol{v} \in \mathbf{H}^{1}(\Omega),
\end{aligned}
$$

Copyright (c) by SIAM. Unauthorized reproduction of this article is prohibited. 
where $\kappa_{1}, \kappa_{2}, \kappa_{3}$, and $\kappa_{4}$ are positive parameters to be specified later. We remark that the identities required in (3.7)-(3.10) are nothing but the constitutive and the equilibrium equations concerning $\boldsymbol{\sigma}$, along with the relation defining $\boldsymbol{t}$ as $\nabla \boldsymbol{u}$, and the Dirichlet condition for the velocity, but all them tested differently from (3.5). We have thus arrived at the following augmented mixed formulation: Find $(\boldsymbol{t}, \boldsymbol{\sigma}, \boldsymbol{u}) \in$ $\mathbf{H}:=\mathbb{L}_{\mathrm{tr}}^{2}(\Omega) \times \mathbb{H}_{0}(\operatorname{div} ; \Omega) \times \mathbf{H}^{1}(\Omega)$ such that

$$
\left[\left(\mathbf{A}+\mathbf{B}_{\boldsymbol{u}}\right)(\boldsymbol{t}, \boldsymbol{\sigma}, \boldsymbol{u}),(\boldsymbol{s}, \boldsymbol{\tau}, \boldsymbol{v})\right]=[\mathbf{F},(\boldsymbol{s}, \boldsymbol{\tau}, \boldsymbol{v})] \quad \forall(\boldsymbol{s}, \boldsymbol{\tau}, \boldsymbol{v}) \in \mathbf{H},
$$

where $[\cdot, \cdot]$ stands for the duality pairing between $\mathbf{H}^{\prime}$ and $\mathbf{H}, \mathbf{A}: \mathbf{H} \longrightarrow \mathbf{H}^{\prime}$ is the nonlinear operator

$$
\begin{aligned}
& {[\mathbf{A}(\boldsymbol{t}, \boldsymbol{\sigma}, \boldsymbol{u}),(\boldsymbol{s}, \boldsymbol{\tau}, \boldsymbol{v})]} \\
& :=\int_{\Omega} \mu(|\boldsymbol{t}|) \boldsymbol{t}: s-\int_{\Omega} \boldsymbol{\sigma}^{\mathrm{d}}: \boldsymbol{s}+\int_{\Omega} \boldsymbol{\tau}^{\mathrm{d}}: \boldsymbol{t} \\
& \quad+\int_{\Omega} \boldsymbol{u} \cdot \operatorname{div} \boldsymbol{\tau}-\int_{\Omega} \boldsymbol{v} \cdot \operatorname{div} \boldsymbol{\sigma}+\kappa_{1} \int_{\Omega}\left\{\boldsymbol{\sigma}^{\mathrm{d}}-\mu(|\boldsymbol{t}|) \boldsymbol{t}\right\}: \boldsymbol{\tau}^{\mathrm{d}} \\
& \quad+\kappa_{2} \int_{\Omega} \operatorname{div} \boldsymbol{\sigma} \cdot \operatorname{div} \boldsymbol{\tau}+\kappa_{3} \int_{\Omega}\{\nabla \boldsymbol{u}-\boldsymbol{t}\}: \nabla \boldsymbol{v}+\kappa_{4} \int_{\Gamma} \boldsymbol{u} \cdot \boldsymbol{v},
\end{aligned}
$$

$\mathbf{F}: \mathbf{H} \longrightarrow \mathrm{R}$ is the bounded linear functional

$$
[\mathbf{F},(\boldsymbol{s}, \boldsymbol{\tau}, \boldsymbol{v})]:=\langle\boldsymbol{\tau} \boldsymbol{\nu}, \boldsymbol{g}\rangle+\int_{\Omega} \boldsymbol{f} \cdot\left\{\boldsymbol{v}-\kappa_{2} \operatorname{div} \boldsymbol{\tau}\right\}+\kappa_{4} \int_{\Gamma} \boldsymbol{g} \cdot \boldsymbol{v},
$$

and for each $\boldsymbol{z} \in \mathbf{H}^{1}(\Omega), \mathbf{B}_{\boldsymbol{z}}: \mathbf{H} \longrightarrow \mathbf{H}^{\prime}$ is the bounded linear operator

$$
\left[\mathbf{B}_{\boldsymbol{z}}(\boldsymbol{t}, \boldsymbol{\sigma}, \boldsymbol{u}),(\boldsymbol{s}, \boldsymbol{\tau}, \boldsymbol{v})\right]:=\int_{\Omega}(\boldsymbol{z} \otimes \boldsymbol{u})^{\mathrm{d}}:\left\{\kappa_{1} \boldsymbol{\tau}^{\mathrm{d}}-\boldsymbol{s}\right\}
$$

for all $(\boldsymbol{t}, \boldsymbol{\sigma}, \boldsymbol{u}),(\boldsymbol{s}, \boldsymbol{\tau}, \boldsymbol{v}) \in \mathbf{H}$. The aforementioned boundedness properties will be confirmed below. Indeed, in the forthcoming sections we study the well-posedness of (3.11) by applying some results on fixed point theory.

3.2. A fixed point approach. We begin the solvability analysis of (3.11) by defining the operator $\mathbf{T}: \mathbf{H}^{1}(\Omega) \longrightarrow \mathbf{H}^{1}(\Omega)$ by

$$
\mathbf{T}(\boldsymbol{z}):=\boldsymbol{u} \quad \forall \boldsymbol{z} \in \mathbf{H}^{1}(\Omega),
$$

where $\boldsymbol{u}$ is the third component of the unique solution (to be confirmed below) of the following nonlinear problem: Find $(\boldsymbol{t}, \boldsymbol{\sigma}, \boldsymbol{u}) \in \mathbf{H}$ such that

$$
\left[\left(\mathbf{A}+\mathbf{B}_{\boldsymbol{z}}\right)(\boldsymbol{t}, \boldsymbol{\sigma}, \boldsymbol{u}),(\boldsymbol{s}, \boldsymbol{\tau}, \boldsymbol{v})\right]=[\mathbf{F},(\boldsymbol{s}, \boldsymbol{\tau}, \boldsymbol{v})] \quad \forall(\boldsymbol{s}, \boldsymbol{\tau}, \boldsymbol{v}) \in \mathbf{H} .
$$

It follows that our augmented mixed formulation (3.11) can be rewritten, equivalently, as the following fixed point problem: Find $\boldsymbol{u} \in \mathbf{H}^{1}(\Omega)$ such that

$$
\mathbf{T}(\boldsymbol{u})=\boldsymbol{u} .
$$

However, we remark in advance that the definition of $\mathbf{T}$ will make sense only in a closed ball of $\mathbf{H}^{1}(\Omega)$.

Now, in order to analyze the well-posedness of (3.15), we first collect a couple of useful inequalities. 
Lemma 3.1. There exists $c_{2}(\Omega)>0$ such that

$$
c_{2}(\Omega)\left\|\boldsymbol{\tau}_{0}\right\|_{0, \Omega}^{2} \leq\left\|\boldsymbol{\tau}^{\mathrm{d}}\right\|_{0, \Omega}^{2}+\|\operatorname{div} \boldsymbol{\tau}\|_{0, \Omega}^{2} \quad \forall \boldsymbol{\tau}=\boldsymbol{\tau}_{0}+c \mathbb{I} \in \mathbb{H}(\operatorname{div} ; \Omega) .
$$

Proof. For the proof see [5, Proposition 3.1, Chapter IV].

Lemma 3.2. There exists $c_{3}(\Omega)>0$ such that

$$
|\boldsymbol{v}|_{1, \Omega}^{2}+\|\boldsymbol{v}\|_{0, \Gamma}^{2} \geq c_{3}(\Omega)\|\boldsymbol{v}\|_{1, \Omega}^{2} \quad \forall \boldsymbol{v} \in \mathbf{H}^{1}(\Omega) .
$$

Proof. For the proof see [24, Lemma 3.3].

In addition, we need to recall from [34] that, under the assumptions given by (2.3), the nonlinear operator induced by $\mu$ is Lipschitz continuous and strongly monotone. More precisely, we have the following result.

LEMmA 3.3. Let $L_{\mu}:=\max \left\{\mu_{2}, 2 \mu_{2}-\mu_{1}\right\}$, where $\mu_{1}$ and $\mu_{2}$ are the bounds of $\mu$ given in (2.3). Then for each $\boldsymbol{r}, \boldsymbol{s} \in \mathbb{L}^{2}(\Omega)$ there holds

$$
\|\mu(|\boldsymbol{r}|) \boldsymbol{r}-\mu(|\boldsymbol{s}|) \boldsymbol{s}\|_{0, \Omega} \leq L_{\mu}\|\boldsymbol{r}-\boldsymbol{s}\|_{0, \Omega}
$$

and

$$
\int_{\Omega}\{\mu(|\boldsymbol{r}|) \boldsymbol{r}-\mu(|\boldsymbol{s}|) \boldsymbol{s}\}:(\boldsymbol{r}-\boldsymbol{s}) \geq \mu_{1}\|\boldsymbol{r}-\boldsymbol{s}\|_{0, \Omega}^{2} .
$$

Proof. See [34, Theorem 3.8] for details.

Then, the following lemma provides sufficient conditions under which the operator $\mathbf{T}$ is well-defined.

LEMma 3.4. Assume that $\kappa_{1} \in\left(0, \frac{2 \delta \mu_{1}}{L_{\mu}}\right)$ and $\kappa_{3} \in\left(0,2 \widetilde{\delta}\left(\mu_{1}-\frac{\kappa_{1} L_{\mu}}{2 \delta}\right)\right)$, with $\delta \in$ $\left(0, \frac{2}{L_{\mu}}\right)$ and $\widetilde{\delta} \in(0,2)$, and that $\kappa_{2}, \kappa_{4}>0$. Then, there exists $\rho_{0}>0$ such that for each $\rho \in\left(0, \rho_{0}\right)$, the problem (3.15) has a unique solution for each $\boldsymbol{z} \in \mathbf{H}^{1}(\Omega)$ such that $\|\boldsymbol{z}\|_{1, \Omega} \leq \rho$. Moreover, there exists a constant $c_{\mathbf{T}}>0$, independent of $\boldsymbol{z}$ and the data $\boldsymbol{f}$ and $\boldsymbol{g}$, such that there holds

$$
\|\mathbf{T}(\boldsymbol{z})\|_{1, \Omega}=\|\boldsymbol{u}\|_{1, \Omega} \leq\|(\boldsymbol{t}, \boldsymbol{\sigma}, \boldsymbol{u})\| \leq c_{\mathbf{T}}\left\{\|\boldsymbol{f}\|_{0, \Omega}+\|\boldsymbol{g}\|_{0, \Gamma}+\|\boldsymbol{g}\|_{1 / 2, \Gamma}\right\} .
$$

Proof. Given $\boldsymbol{z} \in \mathbf{H}^{1}(\Omega)$, we first observe that $\mathbf{A}, \mathbf{B}_{\boldsymbol{z}}$, and hence $\mathbf{A}+\mathbf{B}_{\boldsymbol{z}}$, are Lipschitz continuous. In fact, using the Cauchy-Schwarz inequality, the Lipschitz continuity of the operator induced by $\mu$ (cf. (3.17) in Lemma 3.3), and the trace operator $\gamma_{0}: \mathbf{H}^{1}(\Omega) \longrightarrow \mathbf{L}^{2}(\Gamma)$, we deduce from (3.12) that there exists a positive constant $L_{\mathbf{A}}$, depending on $L_{\mu}$, the parameters $\kappa_{i}, i \in\{1, \ldots, 4\}$, and $\left\|\gamma_{0}\right\|$, such that

$$
[\mathbf{A}(\boldsymbol{t}, \boldsymbol{\sigma}, \boldsymbol{u})-\mathbf{A}(\boldsymbol{r}, \boldsymbol{\zeta}, \boldsymbol{w}),(\boldsymbol{s}, \boldsymbol{\tau}, \boldsymbol{v})] \leq L_{\mathbf{A}}\|(\boldsymbol{t}, \boldsymbol{\sigma}, \boldsymbol{u})-(\boldsymbol{r}, \boldsymbol{\zeta}, \boldsymbol{w})\|\|(\boldsymbol{s}, \boldsymbol{\tau}, \boldsymbol{v})\|
$$

for all $(\boldsymbol{t}, \boldsymbol{\sigma}, \boldsymbol{u}),(\boldsymbol{r}, \boldsymbol{\zeta}, \boldsymbol{w}),(\boldsymbol{s}, \boldsymbol{\tau}, \boldsymbol{v}) \in \mathbf{H}$. In turn, it readily follows from (3.6) and (3.14) that

$$
\begin{aligned}
\left|\left[\mathbf{B}_{\boldsymbol{z}}(\boldsymbol{t}, \boldsymbol{\sigma}, \boldsymbol{u}),(\boldsymbol{s}, \boldsymbol{\tau}, \boldsymbol{v})\right]\right| \leq & \left(\kappa_{1}^{2}+1\right)^{1 / 2}\|\boldsymbol{z}\|_{\mathbf{L}^{4}(\Omega)}\|\boldsymbol{u}\|_{\mathbf{L}^{4}(\Omega)}\|(\boldsymbol{s}, \boldsymbol{\tau})\| \\
\leq & c_{1}(\Omega)\left(\kappa_{1}^{2}+1\right)^{1 / 2}\|\boldsymbol{z}\|_{1, \Omega}\|\boldsymbol{u}\|_{1, \Omega}\|(\boldsymbol{s}, \boldsymbol{\tau})\| \\
\leq & c_{1}(\Omega)\left(\kappa_{1}^{2}+1\right)^{1 / 2}\|\boldsymbol{z}\|_{1, \Omega}\|(\boldsymbol{t}, \boldsymbol{\sigma}, \boldsymbol{u})\|\|(\boldsymbol{s}, \boldsymbol{\tau}, \boldsymbol{v})\| \\
& \forall(\boldsymbol{t}, \boldsymbol{\sigma}, \boldsymbol{u}),(\boldsymbol{s}, \boldsymbol{\tau}, \boldsymbol{v}) \in \mathbf{H}
\end{aligned}
$$

Copyright $@$ by SIAM. Unauthorized reproduction of this article is prohibited. 
which, thanks to the linearity of $\mathbf{B}_{\boldsymbol{z}}$, and together with (3.20), confirms the announced continuity properties. In particular, $L_{\mathbf{A}}+c_{1}(\Omega)\left(\kappa_{1}^{2}+1\right)^{1 / 2}\|\boldsymbol{z}\|_{1, \Omega}$ is the Lipschitzcontinuity constant of $\mathbf{A}+\mathbf{B}_{\boldsymbol{z}}$. Next, it is also clear from (3.12) that

$$
\begin{aligned}
& {[\mathbf{A}(\boldsymbol{r}, \boldsymbol{\zeta}, \boldsymbol{w})-\mathbf{A}(\boldsymbol{s}, \boldsymbol{\tau}, \boldsymbol{v}),(\boldsymbol{r}, \boldsymbol{\zeta}, \boldsymbol{w})-(\boldsymbol{s}, \boldsymbol{\tau}, \boldsymbol{v})]} \\
& =\int_{\Omega}\{\mu(|\boldsymbol{r}|) \boldsymbol{r}-\mu(|\boldsymbol{s}|) \boldsymbol{s}\}:(\boldsymbol{r}-\boldsymbol{s}) \\
& \quad+\kappa_{1}\left\|(\boldsymbol{\zeta}-\boldsymbol{\tau})^{\mathrm{d}}\right\|_{0, \Omega}^{2}-\kappa_{1} \int_{\Omega}\{\mu(|\boldsymbol{r}|) \boldsymbol{r}-\mu(|\boldsymbol{s}|) \boldsymbol{s}\}:(\boldsymbol{\zeta}-\boldsymbol{\tau})^{\mathrm{d}}+\kappa_{2}\|\operatorname{div}(\boldsymbol{\zeta}-\boldsymbol{\tau})\|_{0, \Omega}^{2} \\
& \quad+\kappa_{3}|\boldsymbol{w}-\boldsymbol{v}|_{1, \Omega}^{2}-\kappa_{3} \int_{\Omega}(\boldsymbol{r}-\boldsymbol{s}): \nabla(\boldsymbol{w}-\boldsymbol{v})+\kappa_{4}\|\boldsymbol{w}-\boldsymbol{v}\|_{0, \Gamma}^{2},
\end{aligned}
$$

which, using the Cauchy-Schwarz and Young inequalities, and the Lipschitz continuity and strong monotonicity properties of the operator induced by $\mu$ (cf. (3.17) and (3.18)), yields for any $\delta, \widetilde{\delta}>0$, and for all $(\boldsymbol{r}, \boldsymbol{\zeta}, \boldsymbol{w}),(\boldsymbol{s}, \boldsymbol{\tau}, \boldsymbol{v}) \in \mathbf{H}$,

$$
\begin{aligned}
& {[\mathbf{A}(\boldsymbol{r}, \boldsymbol{\zeta}, \boldsymbol{w})-\mathbf{A}(\boldsymbol{s}, \boldsymbol{\tau}, \boldsymbol{v}),(\boldsymbol{r}, \boldsymbol{\zeta}, \boldsymbol{w})-(\boldsymbol{s}, \boldsymbol{\tau}, \boldsymbol{v})]} \\
& \geq \quad\left\{\left(\mu_{1}-\frac{\kappa_{1} L_{\mu}}{2 \delta}\right)-\frac{\kappa_{3}}{2 \widetilde{\delta}}\right\}\|\boldsymbol{r}-\boldsymbol{s}\|_{0, \Omega}^{2} \\
& \quad+\kappa_{1}\left(1-\frac{L_{\mu} \delta}{2}\right)\left\|(\boldsymbol{\zeta}-\boldsymbol{\tau})^{\mathrm{d}}\right\|_{0, \Omega}^{2}+\kappa_{2}\|\operatorname{div}(\boldsymbol{\zeta}-\boldsymbol{\tau})\|_{0, \Omega}^{2} \\
& \quad+\kappa_{3}\left(1-\frac{\widetilde{\delta}}{2}\right)|\boldsymbol{w}-\boldsymbol{v}|_{1, \Omega}^{2}+\kappa_{4}\|\boldsymbol{w}-\boldsymbol{v}\|_{0, \Gamma}^{2} .
\end{aligned}
$$

Then, assuming the stipulated hypotheses on $\delta, \kappa_{1}, \widetilde{\delta}, \kappa_{3}, \kappa_{2}$, and $\kappa_{4}$, and applying Lemmas 3.1 and 3.2 , we can define the positive constants

$$
\begin{array}{ll}
\alpha_{0}(\Omega):=\left(\mu_{1}-\frac{\kappa_{1} L_{\mu}}{2 \delta}\right)-\frac{\kappa_{3}}{2 \widetilde{\delta}}, & \alpha_{1}(\Omega):=\min \left\{\kappa_{1}\left(1-\frac{L_{\mu} \delta}{2}\right), \frac{\kappa_{2}}{2}\right\}, \\
\alpha_{2}(\Omega):=\min \left\{\alpha_{1}(\Omega) c_{2}(\Omega), \frac{\kappa_{2}}{2}\right\}, & \text { and } \quad \alpha_{3}(\Omega):=c_{3}(\Omega) \min \left\{\kappa_{3}\left(1-\frac{\widetilde{\delta}}{2}\right), \kappa_{4}\right\},
\end{array}
$$

which allow us to deduce from (3.22) that

$$
[\mathbf{A}(\boldsymbol{r}, \boldsymbol{\zeta}, \boldsymbol{w})-\mathbf{A}(\boldsymbol{s}, \boldsymbol{\tau}, \boldsymbol{v}),(\boldsymbol{r}, \boldsymbol{\zeta}, \boldsymbol{w})-(\boldsymbol{s}, \boldsymbol{\tau}, \boldsymbol{v})] \geq \alpha(\Omega)\|(\boldsymbol{r}, \boldsymbol{\zeta}, \boldsymbol{w})-(\boldsymbol{s}, \boldsymbol{\tau}, \boldsymbol{v})\|^{2}
$$

for all $(\boldsymbol{r}, \boldsymbol{\zeta}, \boldsymbol{w}),(\boldsymbol{s}, \boldsymbol{\tau}, \boldsymbol{v}) \in \mathbf{H}$, where

$$
\alpha(\Omega):=\min \left\{\alpha_{0}(\Omega), \alpha_{2}(\Omega), \alpha_{3}(\Omega)\right\}
$$

is the strong monotonicity constant of A. Moreover, by combining (3.21) and (3.23), we obtain

$$
\begin{array}{r}
{\left[\left(\mathbf{A}+\mathbf{B}_{\boldsymbol{z}}\right)(\boldsymbol{r}, \boldsymbol{\zeta}, \boldsymbol{w})-\left(\mathbf{A}+\mathbf{B}_{\boldsymbol{z}}\right)(\boldsymbol{s}, \boldsymbol{\tau}, \boldsymbol{v}),(\boldsymbol{r}, \boldsymbol{\zeta}, \boldsymbol{w})-(\boldsymbol{s}, \boldsymbol{\tau}, \boldsymbol{v})\right]} \\
\quad \geq\left\{\alpha(\Omega)-c_{1}(\Omega)\left(\kappa_{1}^{2}+1\right)^{1 / 2}\|\boldsymbol{z}\|_{1, \Omega}\right\}\|(\boldsymbol{r}, \boldsymbol{\zeta}, \boldsymbol{w})-(\boldsymbol{s}, \boldsymbol{\tau}, \boldsymbol{v})\|^{2} \\
\quad \geq \frac{\alpha(\Omega)}{2}\|(\boldsymbol{r}, \boldsymbol{\zeta}, \boldsymbol{w})-(\boldsymbol{s}, \boldsymbol{\tau}, \boldsymbol{v})\|^{2} \quad \forall(\boldsymbol{r}, \boldsymbol{\zeta}, \boldsymbol{w}),(\boldsymbol{s}, \boldsymbol{\tau}, \boldsymbol{v}) \in \mathbf{H},
\end{array}
$$

Copyright $@$ by SIAM. Unauthorized reproduction of this article is prohibited. 
provided $c_{1}(\Omega)\left(\kappa_{1}^{2}+1\right)^{1 / 2}\|\boldsymbol{z}\|_{1, \Omega} \leq \frac{\alpha(\Omega)}{2}$. Consequently, the strong monotonicity of the nonlinear operator $\mathbf{A}+\mathbf{B}_{\boldsymbol{z}}$ is ensured with the constant $\frac{\alpha(\Omega)}{2}$, independent of $\boldsymbol{z}$, by requiring $\|\boldsymbol{z}\|_{1, \Omega} \leq \rho_{0}$, with

$$
\rho_{0}:=\frac{\alpha(\Omega)}{2 c_{1}(\Omega)\left(\kappa_{1}^{2}+1\right)^{1 / 2}} .
$$

Now, concerning the linear functional $\mathbf{F}$, we readily find from (3.13), by using the Cauchy-Schwarz inequality and the trace theorems in $\mathbb{H}(\mathbf{d i v} ; \Omega)$ and $\mathbf{H}^{1}(\Omega)$, whose boundedness constants are given by 1 and $\left\|\gamma_{0}\right\|$, respectively, that $\mathbf{F} \in \mathbf{H}^{\prime}$ with

$$
\|\mathbf{F}\| \leq M_{\mathbf{T}}\left\{\|\boldsymbol{f}\|_{0, \Omega}+\|\boldsymbol{g}\|_{0, \Gamma}+\|\boldsymbol{g}\|_{1 / 2, \Gamma}\right\}
$$

where $M_{\mathbf{T}}:=\max \left\{\left(1+\kappa_{2}^{2}\right)^{1 / 2}, \kappa_{4}\left\|\gamma_{0}\right\|\right\}$. In this way, having established that the operator $\mathbf{A}+\mathbf{B}_{\boldsymbol{z}}$ is Lipschitz continuous and strongly monotone, and knowing that $\mathbf{F} \in \mathbf{H}^{\prime}$, a classical result on the bijectivity of monotone operators (see, e.g., [38, Theorem 3.3.23]) allows us to conclude that there exists a unique solution $(\boldsymbol{t}, \boldsymbol{\sigma}, \boldsymbol{u}) \in$ $\mathbf{H}$ of (3.15). Finally, by applying (3.24) with $(\boldsymbol{r}, \boldsymbol{\zeta}, \boldsymbol{w})=(\boldsymbol{t}, \boldsymbol{\sigma}, \boldsymbol{u})$ and $(\boldsymbol{s}, \boldsymbol{\tau}, \boldsymbol{v})=$ $(\mathbf{0}, \mathbf{0}, \mathbf{0})$, noting that $\left(\mathbf{A}+\mathbf{B}_{\boldsymbol{z}}\right)(\mathbf{0}, \mathbf{0}, \mathbf{0})$ is the null functional, and performing simple algebraic manipulations, we arrive at (3.19) with the positive constant $c_{\mathbf{T}}:=\frac{2 M_{\mathbf{T}}}{\alpha(\Omega)}$, which is clearly independent of $\boldsymbol{z}$.

We end this section by remarking that the constant $\alpha(\Omega)$ yielding the strong monotonicity of both $\mathbf{A}$ and $\mathbf{A}+\mathbf{B}_{\boldsymbol{z}}$ can be maximized by taking the parameters $\delta$, $\kappa_{1}, \widetilde{\delta}$, and $\kappa_{3}$ as the middle points of their feasible ranges, and by choosing $\kappa_{2}$ and $\kappa_{4}$ so that they maximize the minima defining $\alpha_{1}(\Omega)$ and $\alpha_{3}(\Omega)$, respectively. More precisely, we simply take

$$
\begin{array}{rlrl}
\delta & =\frac{1}{L_{\mu}}, \quad \kappa_{1}=\frac{\delta \mu_{1}}{L_{\mu}}=\frac{\mu_{1}}{L_{\mu}^{2}}, & \widetilde{\delta}=1, & \kappa_{3}=\widetilde{\delta}\left(\mu_{1}-\frac{\kappa_{1} L_{\mu}}{2 \delta}\right)=\frac{\mu_{1}}{2} \\
\kappa_{2}=2 \kappa_{1}\left(1-\frac{L_{\mu} \delta}{2}\right)=\kappa_{1}=\frac{\mu_{1}}{L_{\mu}^{2}}, & \text { and } & \kappa_{4}=\kappa_{3}\left(1-\frac{\widetilde{\delta}}{2}\right)=\frac{\kappa_{3}}{2}=\frac{\mu_{1}}{4}
\end{array}
$$

which yields

$\alpha_{0}(\Omega)=\frac{\mu_{1}}{4}, \quad \alpha_{1}(\Omega)=\frac{\mu_{1}}{2 L_{\mu}^{2}}, \quad \alpha_{2}(\Omega)=\min \left\{c_{2}(\Omega), 1\right\} \frac{\mu_{1}}{2 L_{\mu}^{2}}, \quad \alpha_{3}(\Omega)=c_{3}(\Omega) \frac{\mu_{1}}{4}$,

and hence

$$
\alpha(\Omega)=\min \left\{\min \left\{c_{3}(\Omega), 1\right\} \frac{\mu_{1}}{4}, \min \left\{c_{2}(\Omega), 1\right\} \frac{\mu_{1}}{2 L_{\mu}^{2}}\right\} .
$$

The explicit values of the stabilization parameters $\kappa_{i}, i \in\{1, \ldots, 4\}$, given in (3.26), will be employed in section 5 for the corresponding numerical experiments.

3.3. Solvability analysis of the fixed point equation. We now aim to establish the existence of a unique fixed point of the operator $\mathbf{T}$. To this end, we show next that it suffices to verify the hypotheses of the Schauder fixed point theorem since the uniqueness will follow from the same estimates obtained through that analysis. 
For the sake of completeness, we recall that the aforementioned theorem is stated as follows (see, e.g., [15, Theorem 9.12-1(b)]).

THEOREM 3.5. Let $W$ be a closed and convex subset of a Banach space $X$, and let $T: W \rightarrow W$ be a continuous mapping such that $\overline{T(W)}$ is compact. Then $T$ has at least one fixed point.

We begin the analysis with the following straightforward consequence of Lemma 3.4 .

Lemma 3.6. Let $\rho \in\left(0, \rho_{0}\right)$, with $\rho_{0}$ given by (3.25) (cf. proof of Lemma 3.4), let $W_{\rho}$ be the closed ball defined by $W_{\rho}:=\left\{\boldsymbol{z} \in \mathbf{H}^{1}(\Omega):\|\boldsymbol{z}\|_{1, \Omega} \leq \rho\right\}$, and assume that the data satisfy

$$
c_{\mathbf{T}}\left\{\|\boldsymbol{f}\|_{0, \Omega}+\|\boldsymbol{g}\|_{0, \Gamma}+\|\boldsymbol{g}\|_{1 / 2, \Gamma}\right\} \leq \rho,
$$

with $c_{\mathbf{T}}$ given at the end of the proof of Lemma 3.4. Then there holds $\mathbf{T}\left(W_{\rho}\right) \subseteq W_{\rho}$.

In turn, the following lemma establishes a key estimate to derive next the required continuity and compactness properties of the operator $\mathbf{T}$.

Lemma 3.7. Let $\rho \in\left(0, \rho_{0}\right)$, with $\rho_{0}$ given by $(3.25)$, and let

$$
W_{\rho}:=\left\{\boldsymbol{z} \in \mathbf{H}^{1}(\Omega): \quad\|\boldsymbol{z}\|_{1, \Omega} \leq \rho\right\} .
$$

Then there exists a positive constant $C_{\mathbf{T}}$, depending on $\kappa_{1},\left\|\boldsymbol{i}_{c}\right\|$, and $\alpha(\Omega)$, such that

$$
\|\mathbf{T}(\boldsymbol{z})-\mathbf{T}(\widetilde{\boldsymbol{z}})\|_{1, \Omega} \leq C_{\mathbf{T}}\|\mathbf{T}(\widetilde{\boldsymbol{z}})\|_{1, \Omega}\|\boldsymbol{z}-\widetilde{\boldsymbol{z}}\|_{\mathbf{L}^{4}(\Omega)} \quad \forall \boldsymbol{z}, \widetilde{\boldsymbol{z}} \in W_{\rho} .
$$

Proof. Given $\rho$ as indicated, and $\boldsymbol{z}, \widetilde{\boldsymbol{z}} \in W_{\rho}$, we let $\boldsymbol{u}=\mathbf{T}(\boldsymbol{z})$ and $\widetilde{\boldsymbol{u}}=\mathbf{T}(\widetilde{\boldsymbol{z}})$ be the third components of the corresponding solutions of (3.15), that is

$$
\left[\left(\mathbf{A}+\mathbf{B}_{\boldsymbol{z}}\right)(\boldsymbol{t}, \boldsymbol{\sigma}, \boldsymbol{u}),(\boldsymbol{s}, \boldsymbol{\tau}, \boldsymbol{v})\right]=[\mathbf{F},(\boldsymbol{s}, \boldsymbol{\tau}, \boldsymbol{v})] \quad \forall(\boldsymbol{s}, \boldsymbol{\tau}, \boldsymbol{v}) \in \mathbf{H}
$$

and

$$
\left.\left[\left(\mathbf{A}+\mathbf{B}_{\widetilde{z}}\right) \widetilde{\boldsymbol{t}}, \widetilde{\boldsymbol{\sigma}}, \widetilde{\boldsymbol{u}}\right),(\boldsymbol{s}, \boldsymbol{\tau}, \boldsymbol{v})\right]=[\mathbf{F},(\boldsymbol{s}, \boldsymbol{\tau}, \boldsymbol{v})] \quad \forall(\boldsymbol{s}, \boldsymbol{\tau}, \boldsymbol{v}) \in \mathbf{H} .
$$

Then, applying the strong monotonicity of $\mathbf{A}+\mathbf{B}_{\boldsymbol{z}}$ (cf. (3.24)), we find that

$$
\begin{aligned}
\frac{\alpha(\Omega)}{2} \|(\boldsymbol{t}, \boldsymbol{\sigma}, \boldsymbol{u}) & -(\widetilde{\boldsymbol{t}}, \widetilde{\boldsymbol{\sigma}}, \widetilde{\boldsymbol{u}}) \|^{2} \\
& \leq\left[\left(\mathbf{A}+\mathbf{B}_{\boldsymbol{z}}\right)(\boldsymbol{t}, \boldsymbol{\sigma}, \boldsymbol{u})-\left(\mathbf{A}+\mathbf{B}_{\boldsymbol{z}}\right)(\widetilde{\boldsymbol{t}}, \widetilde{\boldsymbol{\sigma}}, \widetilde{\boldsymbol{u}}),(\boldsymbol{t}, \boldsymbol{\sigma}, \boldsymbol{u})-(\widetilde{\boldsymbol{t}}, \widetilde{\boldsymbol{\sigma}}, \widetilde{\boldsymbol{u}})\right],
\end{aligned}
$$

which, adding and substracting $\mathbf{B}_{\widetilde{\boldsymbol{z}}}(\widetilde{\boldsymbol{t}}, \widetilde{\boldsymbol{\sigma}}, \widetilde{\boldsymbol{u}})$, and then employing (3.29) and (3.30), yields

$$
\frac{\alpha(\Omega)}{2}\|(\boldsymbol{t}, \boldsymbol{\sigma}, \boldsymbol{u})-(\widetilde{\boldsymbol{t}}, \widetilde{\boldsymbol{\sigma}}, \widetilde{\boldsymbol{u}})\|^{2} \leq\left[\mathbf{B}_{\tilde{\boldsymbol{z}}-\boldsymbol{z}}(\widetilde{\boldsymbol{t}}, \widetilde{\boldsymbol{\sigma}}, \widetilde{\boldsymbol{u}}),(\boldsymbol{t}, \boldsymbol{\sigma}, \boldsymbol{u})-(\widetilde{\boldsymbol{t}}, \widetilde{\boldsymbol{\sigma}}, \widetilde{\boldsymbol{u}})\right]
$$

In this way, applying the first estimate in (3.21) to the right-hand side of the foregoing inequality, and then bounding $\|\widetilde{\boldsymbol{u}}\|_{\mathbf{L}^{4}(\Omega)}$ by $\left\|\boldsymbol{i}_{c}\right\|\|\widetilde{\boldsymbol{u}}\|_{1, \Omega}$, we deduce, after a minor 
simplification, that

$$
\|(\boldsymbol{t}, \boldsymbol{\sigma}, \boldsymbol{u})-\widetilde{\boldsymbol{t}}, \widetilde{\boldsymbol{\sigma}}, \widetilde{\boldsymbol{u}})\left\|\leq \frac{2\left(\kappa_{1}^{2}+1\right)^{1 / 2}\left\|\boldsymbol{i}_{c}\right\|}{\alpha(\Omega)}\right\| \widetilde{\boldsymbol{u}}\left\|_{1, \Omega}\right\| \boldsymbol{z}-\widetilde{\boldsymbol{z}} \|_{\mathbf{L}^{4}(\Omega)},
$$

which certainly implies (3.28) with $C_{\mathbf{T}}:=\frac{2\left(\kappa_{1}^{2}+1\right)^{1 / 2}\left\|\boldsymbol{i}_{c}\right\|}{\alpha(\Omega)}$ and completes the proof.

We are now in a position to establish the announced properties of the operator $\mathbf{T}$.

Lemma 3.8. Given $\rho \in\left(0, \rho_{0}\right)$, with $\rho_{0}$ defined by (3.25), we let

$$
W_{\rho}:=\left\{\boldsymbol{z} \in \mathbf{H}^{1}(\Omega):\|\boldsymbol{z}\|_{1, \Omega} \leq \rho\right\},
$$

and assume that the data satisfy (3.27) (cf. Lemma 3.6). Then, $\mathbf{T}: W_{\rho} \longrightarrow W_{\rho}$ is continuous and $\overline{\mathbf{T}\left(W_{\rho}\right)}$ is compact.

Proof. It follows straightforwardly from (3.28) and the continuity of $\boldsymbol{i}_{c}: \mathbf{H}^{1}(\Omega) \longrightarrow$ $\mathbf{L}^{4}(\Omega)$ that

$$
\|\mathbf{T}(\boldsymbol{z})-\mathbf{T}(\widetilde{\boldsymbol{z}})\|_{1, \Omega} \leq C_{\mathbf{T}}\left\|\boldsymbol{i}_{c}\right\|\|\mathbf{T}(\widetilde{\boldsymbol{z}})\|_{1, \Omega}\|\boldsymbol{z}-\widetilde{\boldsymbol{z}}\|_{1, \Omega} \quad \forall \boldsymbol{z}, \widetilde{\boldsymbol{z}} \in W_{\rho},
$$

which proves the continuity of $\mathbf{T}$. Now, given a sequence $\left\{\boldsymbol{z}_{k}\right\}_{k \in \mathrm{N}}$ of $W_{\rho}$, which is clearly bounded, there exists a subsequence $\left\{\boldsymbol{z}_{k}^{(1)}\right\}_{k \in \mathrm{N}} \subseteq\left\{\boldsymbol{z}_{k}\right\}_{k \in \mathrm{N}}$ and $\boldsymbol{z} \in \mathbf{H}^{1}(\Omega)$ such that $\boldsymbol{z}_{k}^{(1)} \stackrel{w}{\longrightarrow} \boldsymbol{z}$ in $\mathbf{H}^{1}(\Omega)$. In this way, thanks to the compactness of $\boldsymbol{i}_{c}$, we deduce that $\boldsymbol{z}_{k}^{(1)} \longrightarrow \boldsymbol{z}$ in $\mathbf{L}^{4}(\Omega)$, which, combined with (3.28), implies that $\mathbf{T}\left(\boldsymbol{z}_{k}^{(1)}\right) \longrightarrow \mathbf{T}(\boldsymbol{z})$ in $\mathbf{H}^{1}(\Omega)$. This proves that $\overline{\mathbf{T}\left(W_{\rho}\right)}$ is compact and finishes the proof.

The main result of this section is stated next.

THEOREM 3.9. Suppose that the parameters $\kappa_{i}, i \in\{1, \ldots, 4\}$, satisfy the conditions required by Lemma 3.4. In addition, given $\rho \in\left(0, \rho_{0}\right)$, with $\rho_{0}$ defined by (3.25), we let $W_{\rho}:=\left\{\boldsymbol{z} \in \mathbf{H}^{1}(\Omega):\|\boldsymbol{z}\|_{1, \Omega} \leq \rho\right\}$, and assume that the data satisfy (3.27) (cf. Lemma 3.6). Then, the augmented mixed formulation (3.11) has a unique solution $(\boldsymbol{t}, \boldsymbol{\sigma}, \boldsymbol{u}) \in \mathbf{H}$ with $\boldsymbol{u} \in W_{\rho}$, and there holds

$$
\|(\boldsymbol{t}, \boldsymbol{\sigma}, \boldsymbol{u})\| \leq c_{\mathbf{T}}\left\{\|\boldsymbol{f}\|_{0, \Omega}+\|\boldsymbol{g}\|_{0, \Gamma}+\|\boldsymbol{g}\|_{1 / 2, \Gamma}\right\} .
$$

Proof. The equivalence between (3.11) and the fixed point equation (3.16), together with Lemmas 3.6 and 3.8, confirms the existence of a solution for (3.11) as a direct application of the Schauder fixed point theorem (cf. Theorem 3.5). In addition, it is clear that the estimate (3.32) follows straightforwardly from (3.19). On the other hand, a second look at the inequality (3.31) and the definitions of the constants $\rho_{0}$ (cf. (3.25)) and $C_{\mathbf{T}}$ (at the end of the proof of Lemma 3.7), give

$$
\|\mathbf{T}(\boldsymbol{z})-\mathbf{T}(\widetilde{\boldsymbol{z}})\|_{1, \Omega} \leq \frac{2\left(\kappa_{1}^{2}+1\right)^{1 / 2} c_{1}(\Omega)}{\alpha(\Omega)}\|\mathbf{T}(\widetilde{\boldsymbol{z}})\|_{1, \Omega}\|\boldsymbol{z}-\widetilde{\boldsymbol{z}}\|_{1, \Omega}=\frac{1}{\rho_{0}}\|\mathbf{T}(\widetilde{\boldsymbol{z}})\|_{1, \Omega}\|\boldsymbol{z}-\widetilde{\boldsymbol{z}}\|_{1, \Omega},
$$

which, thanks again to (3.19), and using our assumption (3.27), implies

$$
\|\mathbf{T}(\boldsymbol{z})-\mathbf{T}(\widetilde{\boldsymbol{z}})\|_{1, \Omega} \leq \frac{1}{\rho_{0}} c_{\mathbf{T}}\left\{\|\boldsymbol{f}\|_{0, \Omega}+\|\boldsymbol{g}\|_{0, \Gamma}+,\|\boldsymbol{g}\|_{1 / 2, \Gamma}\right\}\|\boldsymbol{z}-\widetilde{\boldsymbol{z}}\|_{1, \Omega} \leq \frac{\rho}{\rho_{0}}\|\boldsymbol{z}-\widetilde{\boldsymbol{z}}\|_{1, \Omega}
$$

for all $\boldsymbol{z}, \widetilde{\boldsymbol{z}} \in W_{\rho}$. The foregoing inequality proves that actually, under the hypothesis (3.27), the operator $\mathbf{T}: W_{\rho} \longrightarrow W_{\rho}$ becomes a contraction, and hence it has a unique fixed point. 
Certainly, after seeing the way we proved the previous theorem, we realize that we could have simply applied the Banach fixed point theorem instead of the Schauder's one. However, we prefer to keep the above analysis as it is since, being much more general, it provides a quite useful logical sequence for studying similar and related problems. Indeed, in most of the solvability analyses of more involved fixed point equations, a second condition on the data, different from the one ensuring that the corresponding operator maps a given closed and convex domain into itself, is required for the uniqueness of solutions (see, e.g., [2] for a recent work in this direction concerning a coupled flow-transport problem). The fact that the same condition on the data guarantees both existence and uniqueness of the solution might very well be a particular feature of the present problem and its associated fixed point operator $\mathbf{T}$.

4. The Galerkin scheme. In this section we introduce the Galerkin scheme of the augmented mixed formulation (3.11), analyze its solvability by employing a discrete version of the fixed point strategy developed in section 3.2, and finally derive the corresponding a priori error estimate by applying a suitable Strang-type lemma.

We begin by taking arbitrary finite dimensional subspaces $\mathbb{H}_{h}^{t}, \mathbb{H}_{h}^{\sigma}$, and $\mathbf{H}_{h}^{u}$ of the continuous spaces $\mathbb{L}_{\mathrm{tr}}^{2}(\Omega), \mathbb{H}_{0}(\operatorname{div} ; \Omega)$, and $\mathbf{H}^{1}(\Omega)$, respectively. Hereafter, $h$ denotes the size of a regular triangulation $\mathcal{T}_{h}$ of $\bar{\Omega}$ made up of triangles $K$ (when $n=2$ ) or tetrahedra $K$ (when $n=3$ ) of diameter $h_{K}$, that is $h:=\max \left\{h_{K}: \quad K \in \mathcal{T}_{h}\right\}$. Then, the Galerkin scheme associated with our problem (3.11) reads as follows: Find $\left(\boldsymbol{t}_{h}, \boldsymbol{\sigma}_{h}, \boldsymbol{u}_{h}\right) \in \mathbf{H}_{h}:=\mathbb{H}_{h}^{\boldsymbol{t}} \times \mathbb{H}_{h}^{\boldsymbol{\sigma}} \times \mathbf{H}_{h}^{\boldsymbol{u}}$ such that

$$
\begin{gathered}
{\left[\left(\mathbf{A}+\mathbf{B}_{\boldsymbol{u}_{h}}\right)\left(\boldsymbol{t}_{h}, \boldsymbol{\sigma}_{h}, \boldsymbol{u}_{h}\right),\left(\boldsymbol{s}_{h}, \boldsymbol{\tau}_{h}, \boldsymbol{v}_{h}\right)\right]=\left[\mathbf{F},\left(\boldsymbol{s}_{h}, \boldsymbol{\tau}_{h}, \boldsymbol{v}_{h}\right)\right]} \\
\forall\left(\boldsymbol{s}_{h}, \boldsymbol{\tau}_{h}, \boldsymbol{v}_{h}\right) \in \mathbf{H}_{h} .
\end{gathered}
$$

Next, we let $\mathbf{T}_{h}: \mathbf{H}_{h}^{u} \longrightarrow \mathbf{H}_{h}^{u}$ be the discrete operator defined by

$$
\mathbf{T}_{h}\left(\boldsymbol{z}_{h}\right):=\boldsymbol{u}_{h} \quad \forall \boldsymbol{z}_{h} \in \mathbf{H}_{h}^{u},
$$

where $\boldsymbol{u}_{h}$ is the third component of the unique solution (to be confirmed below) of the following discrete problem: Find $\left(\boldsymbol{t}_{h}, \boldsymbol{\sigma}_{h}, \boldsymbol{u}_{h}\right) \in \mathbf{H}_{h}$ such that

$$
\left[\left(\mathbf{A}+\mathbf{B}_{\boldsymbol{z}_{h}}\right)\left(\boldsymbol{t}_{h}, \boldsymbol{\sigma}_{h}, \boldsymbol{u}_{h}\right),\left(\boldsymbol{s}_{h}, \boldsymbol{\tau}_{h}, \boldsymbol{v}_{h}\right)\right]=\left[\mathbf{F},\left(\boldsymbol{s}_{h}, \boldsymbol{\tau}_{h}, \boldsymbol{v}_{h}\right)\right] \quad \forall\left(\boldsymbol{s}_{h}, \boldsymbol{\tau}_{h}, \boldsymbol{v}_{h}\right) \in \mathbf{H}_{h}
$$

Then, similarly as for the continuous case, it is easy to see that our Galerkin scheme (4.1) can be rewritten, equivalently, as the following fixed point equation: Find $\boldsymbol{u}_{h} \in$ $\mathbf{H}_{h}^{u}$ such that

$$
\mathbf{T}_{h}\left(\boldsymbol{u}_{h}\right)=\boldsymbol{u}_{h}
$$

Now, it is not difficult to see that the arguments employed in the proof of Lemma 3.4 can also be applied to the present discrete setting. In particular, for each $\boldsymbol{z}_{h} \in \mathbf{H}_{h}^{u}$ the nonlinear operator $\mathbf{A}+\mathbf{B}_{\boldsymbol{z}_{h}}: \mathbf{H}_{h} \longrightarrow \mathbf{H}_{h}^{\prime}$ becomes Lipschitz continuous as well with constant $L_{\mathbf{A}}+c_{1}(\Omega)\left(\kappa_{1}^{2}+1\right)^{1 / 2}\left\|\boldsymbol{z}_{h}\right\|_{1, \Omega}$. Moreover, under the same feasible ranges stipulated in Lemma 3.4 for the stabilization parameters and the given $\boldsymbol{z}_{h} \in \mathbf{H}_{h}^{u}$ (instead of $\boldsymbol{z} \in \mathbf{H}^{1}(\Omega)$ ), one finds that $\mathbf{A}+\mathbf{B}_{\boldsymbol{z}_{h}}: \mathbf{H}_{h} \longrightarrow \mathbf{H}_{h}^{\prime}$ becomes strongly monotone with the same constant $\frac{\alpha(\Omega)}{2}$ provided in (3.24). Consequently, the result on monotone operators given by [38, Theorem 3.3.23] implies now the following lemma. 
Lemma 4.1. Assume that $\kappa_{1} \in\left(0, \frac{2 \delta \mu_{1}}{L_{\mu}}\right)$ and $\kappa_{3} \in\left(0,2 \widetilde{\delta}\left(\mu_{1}-\frac{\kappa_{1} L_{\mu}}{2 \delta}\right)\right)$ with $\delta \in$ $\left(0, \frac{2}{L_{\mu}}\right)$ and $\widetilde{\delta} \in(0,2)$, and that $\kappa_{2}, \kappa_{4}>0$. Then, for each $\rho \in\left(0, \rho_{0}\right)$ with $\rho_{0}$ given by (3.25), and for each $\boldsymbol{z}_{h} \in \mathbf{H}_{h}^{\boldsymbol{u}}$ such that $\left\|\boldsymbol{z}_{h}\right\|_{1, \Omega} \leq \rho$, the problem (4.2) has a unique solution $\left(\boldsymbol{t}_{h}, \boldsymbol{\sigma}_{h}, \boldsymbol{u}_{h}\right) \in \mathbf{H}_{h}$. Moreover, with the same constant $c_{\mathbf{T}}>0$ from Lemma 3.4, which is independent of $\boldsymbol{z}_{h}$ and the data $\boldsymbol{f}$ and $\boldsymbol{g}$, there holds

$$
\left\|\mathbf{T}_{h}\left(\boldsymbol{z}_{h}\right)\right\|_{1, \Omega}=\left\|\boldsymbol{u}_{h}\right\|_{1, \Omega} \leq\left\|\left(\boldsymbol{t}_{h}, \boldsymbol{\sigma}_{h}, \boldsymbol{u}_{h}\right)\right\| \leq c_{\mathbf{T}}\left\{\|\boldsymbol{f}\|_{0, \Omega}+\|\boldsymbol{g}\|_{0, \Gamma}+\|\boldsymbol{g}\|_{1 / 2, \Gamma}\right\} .
$$

Moreover, by utilizing the discrete analogue of the analysis developed in section 3.3, we are able to derive the following main result concerning the Galerkin scheme (4.1).

THEOREM 4.2. Suppose that the parameters $\kappa_{i}, i \in\{1, \ldots, 4\}$, satisfy the conditions required by Lemma 4.1. In addition, given $\rho \in\left(0, \rho_{0}\right)$ with $\rho_{0}$ defined by (3.25), we let $W_{\rho}^{h}:=\left\{\boldsymbol{z}_{h} \in \mathbf{H}_{h}^{\boldsymbol{u}}:\left\|\boldsymbol{z}_{h}\right\|_{1, \Omega} \leq \rho\right\}$, and assume that the data satisfy (3.27) (cf. Lemma 3.6), that is

$$
c_{\mathbf{T}}\left\{\|\boldsymbol{f}\|_{0, \Omega}+\|\boldsymbol{g}\|_{0, \Gamma}+\|\boldsymbol{g}\|_{1 / 2, \Gamma}\right\} \leq \rho .
$$

Then, (4.1) has a unique solution $\left(\boldsymbol{t}_{h}, \boldsymbol{\sigma}_{h}, \boldsymbol{u}_{h}\right) \in \mathbf{H}_{h}$ with $\boldsymbol{u}_{h} \in W_{\rho}^{h}$, and there holds

$$
\left\|\left(\boldsymbol{t}_{h}, \boldsymbol{\sigma}_{h}, \boldsymbol{u}_{h}\right)\right\| \leq c_{\mathbf{T}}\left\{\|\boldsymbol{f}\|_{0, \Omega}+\|\boldsymbol{g}\|_{0, \Gamma}+\|\boldsymbol{g}\|_{1 / 2, \Gamma}\right\} .
$$

Proof. We first observe, thanks to (4.4), that the assumption (4.5) guarantees that $\mathbf{T}_{h}\left(W_{\rho}^{h}\right) \subseteq W_{\rho}^{h}$. Next, proceeding as in the proof of Lemma 3.7, that is applying the strong monotonicity of $\mathbf{A}+\mathbf{B}_{\boldsymbol{z}_{h}}: \mathbf{H}_{h} \longrightarrow \mathbf{H}_{h}^{\prime}$ for each $\boldsymbol{z}_{h} \in W_{\rho}^{h}$, and using again the boundedness of the compact injection $\boldsymbol{i}_{c}$ (as at the beginning of the proof of Lemma 3.8), we find that

$$
\left\|\mathbf{T}_{h}\left(\boldsymbol{z}_{h}\right)-\mathbf{T}_{h}\left(\widetilde{\boldsymbol{z}}_{h}\right)\right\|_{1, \Omega} \leq C_{\mathbf{T}}\left\|\boldsymbol{i}_{c}\right\|\left\|\mathbf{T}_{h}\left(\widetilde{\boldsymbol{z}}_{h}\right)\right\|_{1, \Omega}\left\|\boldsymbol{z}_{h}-\widetilde{\boldsymbol{z}}_{h}\right\|_{1, \Omega} \quad \forall \boldsymbol{z}_{h}, \widetilde{\boldsymbol{z}}_{h} \in W_{\rho}^{h},
$$

where $C_{\mathbf{T}}>0$ is the constant introduced in the first aforementioned lemma. Then, employing the same arguments of the proof of Theorem 3.9, in particular using again (4.5), we deduce from the foregoing inequality that

$$
\left\|\mathbf{T}_{h}\left(\boldsymbol{z}_{h}\right)-\mathbf{T}_{h}\left(\widetilde{\boldsymbol{z}}_{h}\right)\right\|_{1, \Omega} \leq \frac{\rho}{\rho_{0}}\left\|\boldsymbol{z}_{h}-\widetilde{\boldsymbol{z}}_{h}\right\|_{1, \Omega} \quad \forall \boldsymbol{z}_{h}, \widetilde{\boldsymbol{z}}_{h} \in W_{\rho}^{h},
$$

which confirms that $\mathbf{T}_{h}: W_{\rho}^{h} \longrightarrow W_{\rho}^{h}$ is also a contraction. In this way, the equivalence between (4.1) and the fixed point equation (4.3) implies the existence of a unique solution of (4.1) as a simple application of the Banach fixed point theorem. In turn, the a priori estimate (4.6) follows directly from (4.4).

Our next goal is to derive an a priori error estimate for our Galerkin scheme (4.1). More precisely, given $\overrightarrow{\boldsymbol{t}}:=(\boldsymbol{t}, \boldsymbol{\sigma}, \boldsymbol{u}) \in \mathbf{H}$, with $\boldsymbol{u} \in W_{\rho}$, and $\overrightarrow{\boldsymbol{t}}_{h}:=\left(\boldsymbol{t}_{h}, \boldsymbol{\sigma}_{h}, \boldsymbol{u}_{h}\right) \in$ $\mathbf{H}_{h}$, with $\boldsymbol{u}_{h} \in W_{\rho}^{h}$, solutions of the problems (3.11) and (4.1), respectively, we are interested in obtaining an upper bound for

$$
\left\|\overrightarrow{\boldsymbol{t}}-\overrightarrow{\boldsymbol{t}}_{h}\right\|=\left\|(\boldsymbol{t}, \boldsymbol{\sigma}, \boldsymbol{u})-\left(\boldsymbol{t}_{h}, \boldsymbol{\sigma}_{h}, \boldsymbol{u}_{h}\right)\right\| .
$$

To this end, we now recall from [29] (see also [2, Lemma 5.1]) a Strang-type lemma that will be utilized in our subsequent analysis. 
Lemma 4.3. Let $\mathbf{H}$ be a Hilbert space, $\mathbf{F} \in \mathbf{H}^{\prime}$, and $\mathbf{S}: \mathbf{H} \rightarrow \mathbf{H}^{\prime}$ a nonlinear operator. In addition, let $\left\{\mathbf{H}_{n}\right\}_{n \in N}$ be a sequence of finite dimensional subspaces of $\mathbf{H}$, and for each $n \in N$ consider a nonlinear operator $\mathbf{S}_{n}: \mathbf{H}_{n} \rightarrow \mathbf{H}_{n}^{\prime}$ and a functional $\mathbf{F}_{n} \in \mathbf{H}_{n}^{\prime}$. Assume that the family $\{\mathbf{S}\} \cup\left\{\mathbf{S}_{n}\right\}_{n \in N}$ is uniformly Lipschitz continuous and strongly monotone with constants $\Lambda_{\mathrm{LC}}$ and $\Lambda_{\mathrm{SM}}$, respectively. In turn, let $u \in \mathbf{H}$ and $u_{n} \in \mathbf{H}_{n}$ such that

$$
[\mathbf{S}(u), v]=[\mathbf{F}, v] \quad \forall v \in \mathbf{H} \quad \text { and } \quad\left[\mathbf{S}_{n}\left(u_{n}\right), v_{n}\right]=\left[\mathbf{F}_{n}, v_{n}\right] \quad \forall v_{n} \in \mathbf{H}_{n},
$$

where $[\cdot, \cdot \cdot]$ denotes the duality pairings of both $\mathbf{H}^{\prime} \times \mathbf{H}$ and $\mathbf{H}_{n}^{\prime} \times \mathbf{H}_{n}$. Then for each $n \in N$ there holds

$$
\begin{aligned}
& \left\|u-u_{n}\right\|_{\mathbf{H}} \\
& \leq \Lambda_{\mathrm{ST}}\left\{\sup _{\substack{w_{n} \in \mathbf{H}_{n} \\
w_{n} \neq \mathbf{0}}} \frac{\left\lfloor\mathbf{F}, w_{n}\right]-\left[\mathbf{F}_{n}, w_{n}\right] \mid}{\left\|w_{n}\right\|_{\mathbf{H}}}\right. \\
& \left.+\inf _{\substack{v_{n} \in \mathbf{H}_{n} \\
v_{n} \neq \mathbf{0}}}\left(\left\|u-v_{n}\right\|_{\mathbf{H}}+\sup _{\substack{w_{n} \in \mathbf{H}_{n} \\
w_{n} \neq \mathbf{0}}} \frac{\left|\left[\mathbf{S}\left(v_{n}\right), w_{n}\right]-\left[\mathbf{S}_{n}\left(v_{n}\right), w_{n}\right]\right|}{\left\|w_{n}\right\|_{\mathbf{H}}}\right)\right\}
\end{aligned}
$$

with $\Lambda_{\mathrm{ST}}:=\Lambda_{\mathrm{SM}}^{-1} \max \left\{1, \Lambda_{\mathrm{SM}}+\Lambda_{\mathrm{LC}}\right\}$.

Proof. The proof is a particular case of [29, Theorem 6.4].

In what follows we apply Lemma 4.3 to the context given by (3.11) and (4.1), which are rewritten as $\overrightarrow{\boldsymbol{t}} \in \mathbf{H}$ and $\overrightarrow{\boldsymbol{t}}_{h} \in \mathbf{H}_{h}$, such that

$$
\left[\left(\mathbf{A}+\mathbf{B}_{u}\right)(\overrightarrow{\boldsymbol{t}}), \overrightarrow{\boldsymbol{s}}\right]=[\mathbf{F}, \overrightarrow{\boldsymbol{s}}] \quad \forall \vec{s} \in \mathbf{H}
$$

and

$$
\left[\left(\mathbf{A}+\mathbf{B}_{\boldsymbol{u}_{h}}\right)\left(\overrightarrow{\boldsymbol{t}}_{h}\right), \overrightarrow{\boldsymbol{s}}_{h}\right]=\left[\mathbf{F}, \overrightarrow{\boldsymbol{s}}_{h}\right] \quad \forall \overrightarrow{\boldsymbol{s}}_{h} \in \mathbf{H}_{h} .
$$

We first notice, thanks to Theorems 3.9 and 4.2, that the Lipschitz continuity constants of $\mathbf{A}+\mathbf{B}_{\boldsymbol{u}}$ and $\mathbf{A}+\mathbf{B}_{\boldsymbol{u}_{h}}$, which are given, respectively, by

$$
L_{\mathbf{A}}+c_{1}(\Omega)\left(\kappa_{1}^{2}+1\right)^{1 / 2}\|\boldsymbol{u}\|_{1, \Omega}
$$

(cf. proof of Lemma 3.4) and $L_{\mathbf{A}}+c_{1}(\Omega)\left(\kappa_{1}^{2}+1\right)^{1 / 2}\left\|\boldsymbol{u}_{h}\right\|_{1, \Omega}$ (cf. remarks right before Lemma 4.1), can be bounded uniformly by the constant

$$
\Lambda_{\mathrm{LC}}:=L_{\mathbf{A}}+c_{1}(\Omega)\left(\kappa_{1}^{2}+1\right)^{1 / 2} \rho_{0}=L_{\mathbf{A}}+\frac{\alpha(\Omega)}{2} .
$$

In turn, it is quite clear from (3.24) and, again, the remarks right before Lemma 4.1, that the strong monotonicity constant of these same nonlinear operators is given by

$$
\Lambda_{\mathrm{SM}}:=\frac{\alpha(\Omega)}{2} .
$$

Consequently, we can prove the following result.

Theorem 4.4. Assume that the data $\boldsymbol{f}$ and $\boldsymbol{g}$ are such that

$$
c_{\mathbf{T}}\left\{\|\boldsymbol{f}\|_{0, \Omega}+\|\boldsymbol{g}\|_{0, \Gamma}+\|\boldsymbol{g}\|_{1 / 2, \Gamma}\right\} \leq \frac{\rho}{2 \max \left\{1, L_{\mathbf{A}}+\alpha(\Omega)\right\}} .
$$

Copyright $@$ by SIAM. Unauthorized reproduction of this article is prohibited. 
Then there exists a positive constant $C$, depending only on $L_{\mathbf{A}}$ and $\alpha(\Omega)$, such that

$$
\left\|\overrightarrow{\boldsymbol{t}}-\overrightarrow{\boldsymbol{t}}_{h}\right\| \leq C \operatorname{dist}\left(\overrightarrow{\boldsymbol{t}}, \mathbf{H}_{h}\right)
$$

Proof. A straightforward application of (4.7) to (4.8)-(4.9) gives

$$
\left\|\overrightarrow{\boldsymbol{t}}-\overrightarrow{\boldsymbol{t}}_{h}\right\|
$$

$$
\leq \Lambda_{\mathrm{ST}} \inf _{\overrightarrow{\boldsymbol{r}}_{h} \in \mathbf{H}_{h}}\left\{\left\|\overrightarrow{\boldsymbol{t}}-\overrightarrow{\boldsymbol{r}}_{h}\right\|+\sup _{\substack{\vec{s}_{h} \in \mathbf{H}_{h} \\ \overrightarrow{\boldsymbol{s}}_{h} \neq \mathbf{0}}} \frac{\left|\left[\left(\mathbf{A}+\mathbf{B}_{\boldsymbol{u}}\right)\left(\overrightarrow{\boldsymbol{r}}_{h}\right), \overrightarrow{\boldsymbol{s}}_{h}\right]-\left[\left(\mathbf{A}+\mathbf{B}_{\boldsymbol{u}_{h}}\right)\left(\overrightarrow{\boldsymbol{r}}_{h}\right), \overrightarrow{\boldsymbol{s}}_{h}\right]\right|}{\left\|\overrightarrow{\boldsymbol{s}}_{h}\right\|}\right\},
$$

where

$$
\Lambda_{\mathrm{ST}}:=\Lambda_{\mathrm{SM}}^{-1} \max \left\{1, \Lambda_{\mathrm{SM}}+\Lambda_{\mathrm{LC}}\right\}=\frac{2}{\alpha(\Omega)} \max \left\{1, L_{\mathbf{A}}+\alpha(\Omega)\right\}
$$

Then, applying the estimate for $\mathbf{B}_{\boldsymbol{z}}$ given by (3.21), adding and substracting $\overrightarrow{\boldsymbol{t}}$, and bounding both $\|\boldsymbol{u}\|_{1, \Omega}$ and $\left\|\boldsymbol{u}_{h}\right\|_{1, \Omega}$ by $\rho_{0}$ at the first term, we find that

$$
\begin{aligned}
& \left|\left[\left(\mathbf{A}+\mathbf{B}_{\boldsymbol{u}}\right)\left(\overrightarrow{\boldsymbol{r}}_{h}\right), \overrightarrow{\boldsymbol{s}}_{h}\right]-\left[\left(\mathbf{A}+\mathbf{B}_{\boldsymbol{u}_{h}}\right)\left(\overrightarrow{\boldsymbol{r}}_{h}\right), \overrightarrow{\boldsymbol{s}}_{h}\right]\right| \\
& \quad=\left|\left[\mathbf{B}_{\boldsymbol{u}-\boldsymbol{u}_{h}}\left(\overrightarrow{\boldsymbol{r}}_{h}\right), \overrightarrow{\boldsymbol{s}}_{h}\right]\right| \leq c_{1}(\Omega)\left(\kappa_{1}^{2}+1\right)^{1 / 2}\left\|\boldsymbol{u}-\boldsymbol{u}_{h}\right\|_{1, \Omega}\left\{\left\|\overrightarrow{\boldsymbol{t}}-\overrightarrow{\boldsymbol{r}}_{h}\right\|+\|\overrightarrow{\boldsymbol{t}}\|\right\}\left\|\overrightarrow{\boldsymbol{s}}_{h}\right\| \\
& \quad \leq\left\{2 c_{1}(\Omega)\left(\kappa_{1}^{2}+1\right)^{1 / 2} \rho_{0}\left\|\overrightarrow{\boldsymbol{t}}-\overrightarrow{\boldsymbol{r}}_{h}\right\|+c_{1}(\Omega)\left(\kappa_{1}^{2}+1\right)^{1 / 2}\|\overrightarrow{\boldsymbol{t}}\|\left\|\boldsymbol{u}-\boldsymbol{u}_{h}\right\|_{1, \Omega}\right\}\left\|\overrightarrow{\boldsymbol{s}}_{h}\right\| \\
& \quad=\left\{\alpha(\Omega)\left\|\overrightarrow{\boldsymbol{t}}-\overrightarrow{\boldsymbol{r}}_{h}\right\|+c_{1}(\Omega)\left(\kappa_{1}^{2}+1\right)^{1 / 2}\|\overrightarrow{\boldsymbol{t}}\|\left\|\boldsymbol{u}-\boldsymbol{u}_{h}\right\|_{1, \Omega}\right\}\left\|\overrightarrow{\boldsymbol{s}}_{h}\right\|,
\end{aligned}
$$

which, replaced back into (4.12), taking the infimum, and using that $\left\|\boldsymbol{u}-\boldsymbol{u}_{h}\right\|_{1, \Omega} \leq$ $\left\|\overrightarrow{\boldsymbol{t}}-\overrightarrow{\boldsymbol{t}}_{h}\right\|$, yields

$$
\left\|\overrightarrow{\boldsymbol{t}}-\overrightarrow{\boldsymbol{t}}_{h}\right\| \leq \Lambda_{\mathrm{ST}}\{1+\alpha(\Omega)\} \operatorname{dist}\left(\overrightarrow{\boldsymbol{t}}, \mathbf{H}_{h}\right)+\Lambda_{\mathrm{ST}} c_{1}(\Omega)\left(\kappa_{1}^{2}+1\right)^{1 / 2}\|\overrightarrow{\boldsymbol{t}}\|\left\|\overrightarrow{\boldsymbol{t}}-\overrightarrow{\boldsymbol{t}}_{h}\right\| .
$$

Finally, recalling from (3.32) that $\|\overrightarrow{\boldsymbol{t}}\| \leq c_{\mathbf{T}}\left\{\|\boldsymbol{f}\|_{0, \Omega}+\|\boldsymbol{g}\|_{0, \Gamma}+\|\boldsymbol{g}\|_{1 / 2, \Gamma}\right\}$, employing our assumption (4.10), and replacing the expression of $\Lambda_{\mathrm{ST}}$ given by (4.13), we obtain that

$$
\Lambda_{\mathrm{ST}} c_{1}(\Omega)\left(\kappa_{1}^{2}+1\right)^{1 / 2}\|\overrightarrow{\boldsymbol{t}}\| \leq \frac{1}{2}
$$

which, together with (4.14), implies (4.11) with $C=2 \Lambda_{\mathrm{ST}}\{1+\alpha(\Omega)\}$, thus completing the proof.

Having established the previous theorem, we now aim to estimate the error for the postprocessed pressure. In fact, according to the second equation of (2.7), and 
(3.3), we define our discrete approximation of the pressure as

$p_{h}:=-\frac{1}{n} \operatorname{tr}\left\{\boldsymbol{\sigma}_{h}+c_{h} \mathbb{I}+\left(\boldsymbol{u}_{h} \otimes \boldsymbol{u}_{h}\right)\right\} \quad$ in $\quad \Omega, \quad$ with $\quad c_{h}:=-\frac{1}{n|\Omega|} \int_{\Omega} \operatorname{tr}\left(\boldsymbol{u}_{h} \otimes \boldsymbol{u}_{h}\right)$,

which yields

$$
p-p_{h}=\frac{1}{n} \operatorname{tr}\left\{\left(\boldsymbol{\sigma}_{h}-\boldsymbol{\sigma}\right)+\left(\boldsymbol{u}_{h} \otimes \boldsymbol{u}_{h}-\boldsymbol{u} \otimes \boldsymbol{u}\right)\right\}+\left(c_{h}-c\right),
$$

and thus, applying the Cauchy-Schwarz inequality, we first find that

$$
\left\|p-p_{h}\right\|_{0, \Omega} \leq \widehat{C}\left\{\left\|\boldsymbol{\sigma}-\boldsymbol{\sigma}_{h}\right\|_{0, \Omega}+\left\|\boldsymbol{u}_{h} \otimes \boldsymbol{u}_{h}-\boldsymbol{u} \otimes \boldsymbol{u}\right\|_{0, \Omega}+\left|c-c_{h}\right|\right\},
$$

where $\widehat{C}>0$ depends on $n$ and $|\Omega|$. Next, bearing in mind the expression for $c$ given by (3.3), decomposing

$$
\boldsymbol{u}_{h} \otimes \boldsymbol{u}_{h}-\boldsymbol{u} \otimes \boldsymbol{u}=\left(\boldsymbol{u}_{h}-\boldsymbol{u}\right) \otimes \boldsymbol{u}_{h}+\boldsymbol{u} \otimes\left(\boldsymbol{u}_{h}-\boldsymbol{u}\right),
$$

and employing the triangle and Hölder inequalities, the compact embedding $\boldsymbol{i}_{c}$ : $\mathbf{H}^{1}(\Omega) \longrightarrow \mathbf{L}^{4}(\Omega)$, and the a priori bounds for $\|\boldsymbol{u}\|_{1, \Omega}$ and $\left\|\boldsymbol{u}_{h}\right\|_{1, \Omega}$ (cf. (3.32) in Theorem 3.9 and (4.6) in Theorem 4.2), we deduce from the foregoing equations that there exists a constant $C>0$, depending on $n,|\Omega|, c_{1}(\Omega)=\left\|\boldsymbol{i}_{c}\right\|^{2}$, and the data $\boldsymbol{f}$ and $\boldsymbol{g}$, but independent of $h$, such that

$$
\left\|p-p_{h}\right\|_{0, \Omega} \leq C\left\{\left\|\boldsymbol{\sigma}-\boldsymbol{\sigma}_{h}\right\|_{\operatorname{div} ; \Omega}+\left\|\boldsymbol{u}-\boldsymbol{u}_{h}\right\|_{1, \Omega}\right\} .
$$

We end this section by defining specific finite element subspaces $\mathbb{H}_{h}^{t}, \mathbb{H}_{h}^{\sigma}$, and $\mathbf{H}_{h}^{u}$, and providing the corresponding rate of convergence of the associated Galerkin scheme (4.1). In what follows, given an integer $k \geq 0$ and a set $\mathrm{S} \subseteq \mathrm{R}^{n}, \mathrm{P}_{k}(\mathrm{~S})$ denotes the space of polynomial functions on $\mathrm{S}$ of degree $\leq k$. In addition, according to the notation described in section 2.1, we set $\mathbf{P}_{k}(S):=\left[\mathrm{P}_{k}(S)\right]^{n}$ and $\mathbb{P}_{k}(S):=\left[\mathrm{P}_{k}(S)\right]^{n \times n}$. Similarly, $\mathbf{C}(\bar{\Omega})=[C(\bar{\Omega})]^{n}$. Then, we introduce the finite element subspaces approximating the unknowns $\boldsymbol{t}$ and $\boldsymbol{u}$ as the piecewise polynomial tensors of degree $\leq k$, and the continuous piecewise polynomial vectors of degree $\leq k+1$, respectively, that is

$$
\mathbb{H}_{h}^{t}:=\left\{\boldsymbol{s}_{h} \in \mathbb{L}_{\mathrm{tr}}^{2}(\Omega):\left.\quad \boldsymbol{s}_{h}\right|_{K} \in \mathbb{P}_{k}(K) \quad \forall K \in \mathcal{T}_{h}\right\}
$$

and

$$
\mathbf{H}_{h}^{u}:=\left\{\boldsymbol{v}_{h} \in \mathbf{C}(\bar{\Omega}):\left.\quad \boldsymbol{v}_{h}\right|_{K} \in \mathbf{P}_{k+1}(K) \quad \forall K \in \mathcal{T}_{h}\right\} .
$$

In turn, for each $K \in \mathcal{T}_{h}$ we set the local Raviart-Thomas space of order $k$ as

$$
\mathbf{R T}_{k}(K):=\mathbf{P}_{k}(K) \oplus \mathrm{P}_{k}(K) \boldsymbol{x},
$$

where $\boldsymbol{x}$ is a generic vector in $\mathrm{R}^{n}$, and define the finite element subspace approximating $\boldsymbol{\sigma}$ as the global Raviart-Thomas space of order $k$, that is

$$
\mathbb{H}_{h}^{\boldsymbol{\sigma}}:=\left\{\boldsymbol{\tau}_{h} \in \mathbb{H}_{0}(\operatorname{div} ; \Omega):\left.\quad \boldsymbol{c}^{t} \boldsymbol{\tau}\right|_{K} \in \mathbf{R T}_{k}(K), \quad \forall \boldsymbol{c} \in \mathrm{R}^{n} \quad \forall K \in \mathcal{T}_{h}\right\} .
$$

The approximation properties of the above finite element subspaces are as follows (cf. $[5,27])$ : 
$\left(\mathbf{A P}_{h}^{t}\right)$ there exists $C>0$, independent of $h$, such that for each $s \in(0, k+1]$, and for each $\boldsymbol{r} \in \mathbb{H}^{s}(\Omega) \cap \mathbb{L}_{\mathrm{tr}}^{2}(\Omega)$, there holds

$$
\operatorname{dist}\left(\boldsymbol{r}, \mathbb{H}_{h}^{t}\right) \leq C h^{s}\|\boldsymbol{r}\|_{s, \Omega} ;
$$

$\left(\mathbf{A P}_{h}^{\sigma}\right)$ there exists $C>0$, independent of $h$, such that for each $s \in(0, k+1]$, and for each $\zeta \in \mathbb{H}^{s}(\Omega) \cap \mathbb{H}_{0}(\operatorname{div} ; \Omega)$ with $\operatorname{div} \zeta \in \mathbf{H}^{s}(\Omega)$, there holds

$$
\operatorname{dist}\left(\boldsymbol{\zeta}, \mathbb{H}_{h}^{\boldsymbol{\sigma}}\right) \leq C h^{s}\left\{\|\boldsymbol{\zeta}\|_{s, \Omega}+\|\operatorname{div} \boldsymbol{\zeta}\|_{s, \Omega}\right\} ;
$$

$\left(\mathbf{A P}_{h}^{u}\right)$ there exists $C>0$, independent of $h$, such that for each $s \in(0, k+1]$, and for each $\boldsymbol{w} \in \mathbf{H}^{s+1}(\Omega)$, there holds

$$
\operatorname{dist}\left(\boldsymbol{w}, \mathbf{H}_{h}^{\boldsymbol{u}}\right) \leq C h^{s}\|\boldsymbol{w}\|_{s+1, \Omega} .
$$

We are now in a position to establish the rate of convergence of the Galerkin scheme (4.1) when the specific finite element subspaces given by (4.17), (4.18), and (4.19), are utilized. We notice here that the main assumption on the data guaranteeing the well-posedness of the continuous and discrete schemes, which is given by (3.27), follows from (4.10), and hence it suffices to assume the latter only.

Theorem 4.5. Besides the hypotheses of Lemma 4.1 (or Lemma 3.4) and Theorem 4.4, assume that there exists $s>0$ such that $\boldsymbol{t} \in \mathbb{H}^{s}(\Omega), \boldsymbol{\sigma} \in \mathbb{H}^{s}(\Omega)$, $\operatorname{div} \boldsymbol{\sigma} \in$ $\mathbf{H}^{s}(\Omega)$, and $\boldsymbol{u} \in \mathbf{H}^{s+1}(\Omega)$, and that the finite element subspaces are defined by (4.17)(4.19). Then, there exists $C>0$, independent of $h$, such that for each $h>0$ there holds

$$
\left\|\overrightarrow{\boldsymbol{t}}-\overrightarrow{\boldsymbol{t}}_{h}\right\|+\left\|p-p_{h}\right\|_{0, \Omega} \leq C h^{\min \{s, k+1\}}\left\{\|\boldsymbol{t}\|_{s, \Omega}+\|\boldsymbol{\sigma}\|_{s, \Omega}+\|\operatorname{div} \boldsymbol{\sigma}\|_{s, \Omega}+\|\boldsymbol{u}\|_{s+1, \Omega}\right\} .
$$

Proof. The proof follows from the Céa estimate (4.11), the upper bound given by (4.16), and the approximation properties $\left(\mathbf{A} \mathbf{P}_{h}^{t}\right),\left(\mathbf{A P}_{h}^{\sigma}\right)$, and $\left(\mathbf{A} \mathbf{P}_{h}^{u}\right)$.

5. Numerical results. In this section we present two examples illustrating the performance of our augmented mixed finite element scheme (4.1), and illustrating the rates of convergence provided by Theorem 4.5. In agreement with (3.26), both tests of this section use the stabilization parameters $\kappa_{1}=\mu_{1} / L_{\mu}^{2}$, with $L_{\mu}:=$ $\max \left\{\mu_{2}, 2 \mu_{2}-\mu_{1}\right\}, \kappa_{2}=\kappa_{1}, \kappa_{3}=\mu_{1} / 2, \kappa_{4}=\mu_{1} / 4$. In addition, the null mean value of $\operatorname{tr} \sigma_{h}$ over $\Omega$ is fixed via a penalization strategy. A Newton algorithm with a tolerance of $1 \mathrm{E}-6$ on the energy norm of the residual has been employed to linearize (4.1).

In our first numerical test we take the unit square as computational domain $\Omega=(0,1)^{2}$, set the nonlinear viscosity to

$$
\mu(s):=2+\frac{1}{1+s} \quad \text { for } s \geq 0,
$$

and construct a series of successively refined triangulations. The accuracy of the method is assessed by choosing the following smooth manufactured exact solution to (3.11):

$$
\begin{aligned}
& p=x_{1}^{2}-x_{2}^{2}, \quad \boldsymbol{u}=\left(\begin{array}{c}
-\cos \left(\pi x_{1}\right) \sin \left(\pi x_{2}\right) \\
\sin \left(\pi x_{1}\right) \cos \left(\pi x_{2}\right)
\end{array}\right), \quad \boldsymbol{t}=\nabla \boldsymbol{u}, \\
& \boldsymbol{\sigma}=\mathbb{H}_{0}(\operatorname{div} ; \Omega) \text {-component of } \mu(|\nabla \boldsymbol{u}|) \nabla \boldsymbol{u}-(\boldsymbol{u} \otimes \boldsymbol{u})-p \mathbb{I},
\end{aligned}
$$

Copyright $@$ by SIAM. Unauthorized reproduction of this article is prohibited. 
TABLE 1

Example 1: Convergence history and Newton iteration count for the mixed-primal $\mathbb{P}_{k}-\mathbb{R} \mathbb{T}_{k}-$ $\mathbf{P}_{k+1}$ approximations of the coupled problem and convergence of the $P_{k}$-approximation of the postprocessed pressure field. Values for $k=0,1$.

\begin{tabular}{|c|c|c|c|c|c|c|c|c|c|c|}
\hline dof & $h$ & $e(\boldsymbol{t})$ & $r(\boldsymbol{t})$ & $e(\boldsymbol{\sigma})$ & $r(\boldsymbol{\sigma})$ & $e(\boldsymbol{u})$ & $r(\boldsymbol{u})$ & $e(p)$ & $r(p)$ & iter \\
\hline \multicolumn{11}{|c|}{ Augmented $\mathbb{P}_{0}-\mathbb{R T}_{0}-\mathbf{P}_{1}$ scheme } \\
\hline 74 & 707 & 1.507 & - & 15.4 & - & 2.13 & - & 1.47 & - & 4 \\
\hline 152 & 0 & 1.032 & 0.934676 & 10.7 & 0.886945 & 1.54 & 0.80 & 1.1 & 0.716 & A \\
\hline 392 & 82843 & 0.627 & 0.974346 & 6.642553 & 0.950075 & 0.966928 & 0.9 & 0.7 & 35 & 5 \\
\hline 1208 & 0.157135 & 0.349 & 0.994165 & 3.738210 & 0.978058 & 0.545122 & 0.9 & 0.40 & 0.954784 & 5 \\
\hline 4184 & 0.083 & 0.18 & 51 & 1.992707 & 0.989188 & 0.2 & 0.9 & 0.2 & 0.991792 & 4 \\
\hline 2 & 0.042855 & 0.09 & 99769 & 1.030370 & 0.994395 & 0.1 & 52 & 0. & 1.001313 & 4 \\
\hline 59672 & 0.021757 & 0.04 & 0.999951 & 0.52 & 0.997430 & $0.0^{\prime}$ & $0 . s$ & 0. & 1.002313 & 4 \\
\hline & 0.010963 & 0.0 & 989 & 0.26 & 0.998 & 0.0 & & & 1.001622 & 3 \\
\hline \multicolumn{11}{|c|}{ Augmented $\mathbb{P}_{1}-\mathbb{R T}_{1}-\mathbf{P}_{2}$ scheme } \\
\hline & & & - & 5.9 & - & 0.7 & - & & - & 3 \\
\hline & 0 & 0.2 & 1.660 & 2.4 & 1.76 & & & & 31 & 4 \\
\hline & 0.28 & 0.0 & 2.10 & 1.0 & 1.66 & & 2.0 & & 2.006908 & 4 \\
\hline 4 & 0.157 & 0.02 & 2.071445 & 0.4 & 1.7 & 0.0 & $2 .($ & & 2.098233 & 4 \\
\hline & 083 & 0.0 & 2.025738 & 0.1 & 1.984209 & 0.0 & 2.003165 & 0.0 & 2.040821 & 5 \\
\hline 40 & 0.042855 & 0.00 & 2.007674 & 0.05 & 1.921534 & 0.0 & 1.997942 & 0.0 & 2.009621 & 5 \\
\hline & 0.0217 & 0.00 & 2.002121 & 0.01 & 1.985 & & 1.997 & 0.00 & 2.001882 & 5 \\
\hline 518714 & 0.010963 & 0.000121 & 2.001908 & 0.00 & 1.993961 & 0.000163 & $1.99939^{\prime}$ & 0.000078 & 1.999572 & 4 \\
\hline
\end{tabular}

and the load function $\boldsymbol{f}$ along with the boundary data $\boldsymbol{g}$ are chosen according to these solutions. In turn, errors and convergence rates are defined as usual:

$$
\begin{gathered}
e(\boldsymbol{t})=\left\|\boldsymbol{t}-\boldsymbol{t}_{h}\right\|_{0, \Omega}, \quad e(\boldsymbol{\sigma})=\left\|\boldsymbol{\sigma}-\boldsymbol{\sigma}_{h}\right\|_{\text {div } ; \Omega}, \quad e(\boldsymbol{u})=\left\|\boldsymbol{u}-\boldsymbol{u}_{h}\right\|_{1, \Omega}, \\
e(p)=\left\|p-p_{h}\right\|_{0, \Omega}, \quad r(\cdot)=\frac{\log (e(\cdot) / \widehat{e}(\cdot))}{\log (h / \widehat{h})}
\end{gathered}
$$

where $e$ and $\widehat{e}$ denote errors computed on two consecutive meshes of sizes $h$ and $\widehat{h}$.

From Table 1 we observe that as the mesh is refined, optimal convergences are attained for the velocity gradient $\boldsymbol{t}$, the pseudostress tensor $\boldsymbol{\sigma}$, and the velocity $\boldsymbol{u}$, that is, the proposed augmented method achieves $O\left(h^{k+1}\right)$ convergence (with $k=0$ and $k=1$ ) for all fields in their relevant norms (as predicted by Theorem 4.5), and around five Newton iterations are required to reach the desired tolerance. Of course, for a fixed mesh, the augmented method corresponding to $k=1$ delivers smaller errors than those generated with $k=0$. All components of the numerical solutions obtained at the finest level are portrayed in Figure 1. At each iteration the resulting linear systems were solved with the multifrontal direct solver MUMPS.

Next, in order to assess the feasibility of the three dimensional implementation, we carry out an extension of the flow over a backward-facing step test performed in [3] (see also [4]). The domain consists of a channel of width 1, height 2, length 6 , and having a step located at the inlet, of height 1 and length 1 (in dimensionless units). The external force is set to zero and the three dimensional flow patterns are determined by the shape of the domain and by the boundary conditions: At the outflow boundary $\left(x_{1}=5\right)$ we set the mean value of the (pseudo)stress to zero, we consider a Poiseuille inflow profile (imposed as a Dirichlet velocity datum at the inlet, $x_{1}=-1$ ), and the remainder of $\partial \Omega$ are treated as rigid walls (putting no-slip 

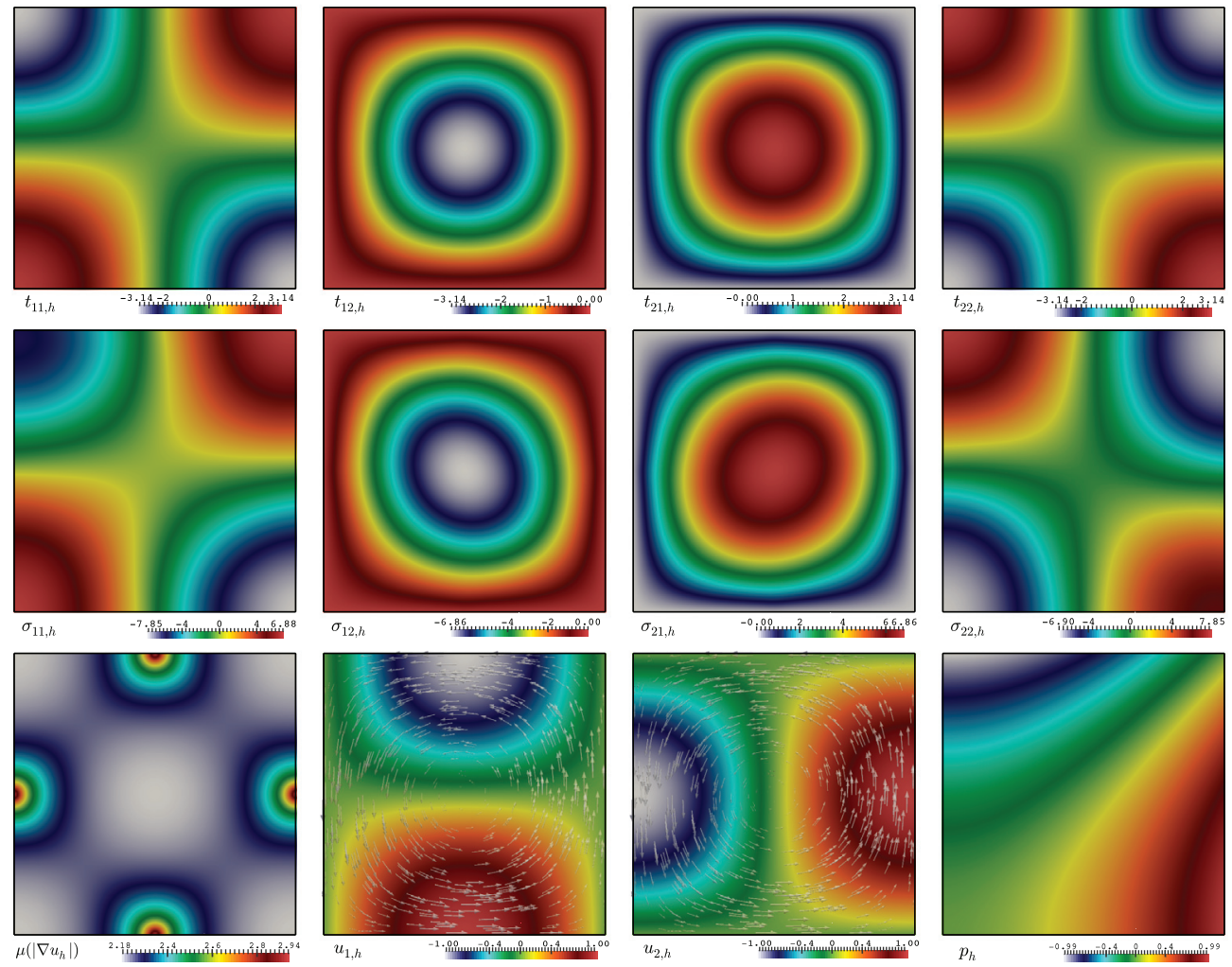

Fig. 1. Test $1: \mathbb{P}_{1}-\mathbb{R T}_{1}-\mathbf{P}_{2}$ approximation of velocity gradient components (top panels), pseudostress components (center panels), and viscosity, velocity components with vector directions, and postprocessed pressure field (bottom row).

velocities). We have

$$
\boldsymbol{g}:= \begin{cases}\left(400 x_{x}\left(1-x_{2}\right) x_{3}^{3}\left(1-x_{3}\right)^{3}, 0,0\right)^{T} & \text { on the inlet } x_{1}=-1, \\ \mathbf{0} & \text { on the rigid walls. }\end{cases}
$$

The viscosity now follows a Carreau law (cf. (2.4)) with $\alpha_{0}=3 / 200, \alpha_{1}=1 / 20$, and $\beta=1$, that is

$$
\mu(s):=\frac{3}{200}+\frac{1}{20}\left(1+s^{2}\right)^{-1 / 2} \text { for } s \geq 0,
$$

which yields a Reynolds number up to 200 , and we construct an unstructured tetrahedral mesh of 24710 vertices and 114504 elements representing a total of 1709892 degrees of freedom for the lowest-order finite element family $k=0$. In combination with the Newton solve, a homotopy method was applied on the viscosity parameter $\alpha_{1}$. Here a BICGSTAB method with left Schur complement preconditionning was used to solve the resulting linear systems, and six Newton steps were needed to achieve the given tolerance. The approximate solutions are depicted in Figure 2. As expected, a smooth flow behavior occurs away from the step, whereas a recirculation zone forms right after the reentrant corner. In addition, here the nonlinear viscosity produces a singular behavior on the pseudostress components. 

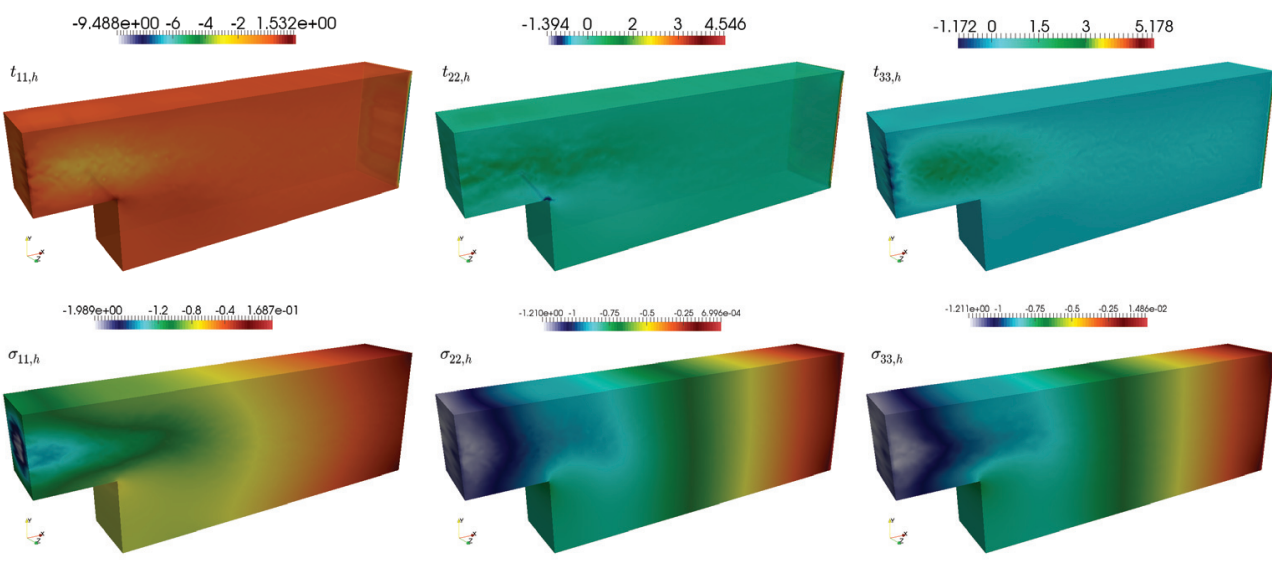

-6.677 e-020.2 $\quad 0.4 \quad 0.6 \quad 9.921$ e-01

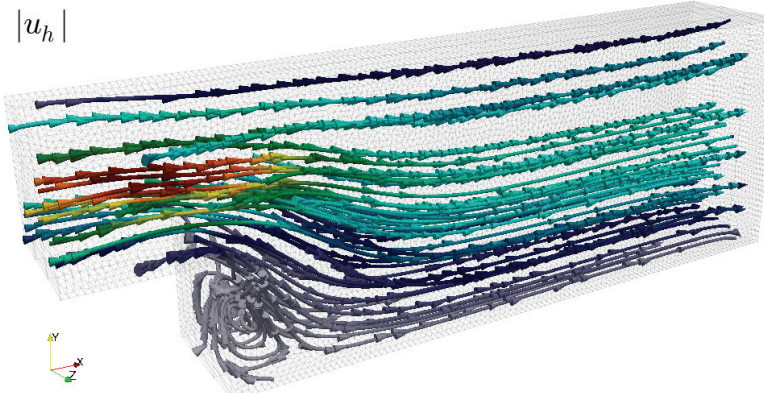

Fig. 2. Test 2: Numerical solutions (diagonal strain and stress components, velocity streamlines, and vector directions) for the flow over a step benchmark using $\mathbb{P}_{0}-\mathbb{R}_{0}-\mathbf{P}_{1}$ approximations of velocity gradient, Cauchy stress, and velocity.

Acknowledgment. The authors are thankful to Ricardo Ruiz-Baier (Mathematical Institute, University of Oxford) for performing the code and running the numerical experiments provided in section 5 .

\section{REFERENCES}

[1] R.A. Adams And J.J.F. Fournier, Sobolev Spaces, 2nd ed., Pure Appl. Math., 140, Elsevier, Amsterdam, 2003.

[2] M. Álvarez, G.N. Gatica, and R. Ruiz-Baier, An augmented mixed-primal finite element method for a coupled flow-transport problem, ESAIM Math. Model. Numer. Anal. 49 (2015), pp. 1399-1427.

[3] V. Anaya, D. Mora, R. Oyarzúa, And R. Ruiz-Baier, A priori and a posteriori error analysis of a mixed scheme for the Brinkman problem, Numer. Math., to appear.

[4] M. Benzi, M. A. Olshanskit, L. G. Rebholz, and Z. Wang, Assessment of a vorticity based solver for the Navier-Stokes equations, Comput. Methods Appl. Mech. Engrg., 247-248 (2012), pp. 216-225.

[5] F. Brezzi And M. Fortin, Mixed and Hybrid Finite Element Methods, Springer Ser. Comput. Math. 15, Springer-Verlag, New York, 1991.

[6] F. BrezzI, J. RAPPAz, AND P.-A. RAVIART, Finite-dimensional approximation of nonlinear problems. I. Branches of nonsingular solutions, Numer. Math., 36 (1980), pp. 1-25.

[7] Z. CAI, B. LEE, AND P. WANG, Least-squares methods for incompressible Newtonian fluid flow: Linear stationary problems, SIAM J. Numer. Anal., 42 (2004), pp. 843-859. 
[8] Z. Cai And G. Starke, Least-squares methods for linear elasticity, SIAM J. Numer. Anal., 42 (2004), pp. 826-842.

[9] Z. Cai, C. Tong, P.S. Vassilevski, and C. Wang, Mixed finite element methods for incompressible flow: Stationary Stokes equations, Numer. Methods Partial Differential Equations, 26 (2010), pp. 957-978.

[10] Z. CAI, C. WAng, AND S. Zhang, Mixed finite element methods for incompressible flow: Stationary Navier-Stokes equations, SIAM J. Numer. Anal., 48 (2010), pp. 79-94.

[11] Z. CAi AND Y. WAng, Pseudostress-velocity formulation for incompressible Navier-Stokes equations, Internat. J. Numer. Methods Fluids, 63 (2010), pp. 341-356.

[12] Z. CAI AND S. ZHANG, Mixed methods for stationary Navier-Stokes equations based on pseudostress-pressure-velocity formulation, Math. Comp., 81 (2012), pp. 1903-1927.

[13] J. Camaño, R. Oyarzúa, and G. Tierra, Analysis of an augmented mixed-FEM for the Navier-Stokes problem, Math. Comp., to appear.

[14] E. Castillo And R. Codina, Stabilized stress-velocity-pressure finite element formulations of the Navier-Stokes problem for fluids with non-linear viscosity, Comput. Methods Appl. Mech. Engrg., 279 (2014), pp. 554-578.

[15] P. Ciarlet, Linear and Nonlinear Functional Analysis with Applications, Appl. Math. 130, SIAM, Philadelphia, 2013.

[16] E. Colmenares, G.N. Gatica, and R. Oyarzúa, Analysis of an augmented mixed-primal formulation for the stationary Boussinesq problem, Numer. Methods Partial Differential Equations, 32 (2016), pp. 445-478, doi:10.1002/num.22001.

[17] V.J. ERvin, J.S. Howell, and I. Stanculescu, A dual-mixed approximation method for a three-field model of a nonlinear generalized Stokes problem, Comput. Methods Appl. Mech. Engrg., 197 (2008), pp. 2886-2900.

[18] V.J. Ervin, E.W. Jenkins, And S. Sun, Coupled generalized nonlinear Stokes flow with flow through a porous medium, SIAM J. Numer. Anal., 47 (2009), pp. 929-952.

[19] V.J. Ervin, E.W. Jenkins, And S. Sun, Coupling nonlinear Stokes and Darcy flow using mortar finite elements, Appl. Numer. Math., 61 (2011), pp. 1198-1222.

[20] M. Farhloul AND H. ManouzI, Analysis of non-singular solutions of a mixed Navier-Stokes formulation, Comput. Methods Appl. Mech. Engrg., 129 (1996), pp. 115-131.

[21] M. Farhloul, S. Nicaise, and L. Paquet, A priori and a posteriori error estimations for the dual mixed finite element method of the Navier-Stokes problem, Numer. Methods Partial Differential Equations, 25 (2009), pp. 843-869.

[22] M. Farhloul AND A.M. Zine, A mixed finite element method for a Ladyzhenskaya model, Comput. Methods Appl. Mech. Engrg., 191 (2002), pp. 4497-4510.

[23] L. Figueroa, G.N. Gatica, and N. Heuer, A priori and a posteriori error analysis of an augmented mixed finite element method for incompressible fluid flows, Comput. Methods Appl. Mech. Engrg., 198 (2008), pp. 280-291.

[24] L.E. Figueroa, G.N. Gatica, and A. Márquez, Augmented mixed finite element methods for the stationary Stokes equations, SIAM J. Sci. Comput., 31 (2008), pp. 1082-1119.

[25] G.N. Gatica, Analysis of a new augmented mixed finite element method for linear elasticity allowing $\mathbb{R T}_{0}-\mathbb{P}_{1}-\mathbb{P}_{0}$ approximations, ESAIM Math. Model. Numer. Anal., 40 (2006), pp. $1-28$.

[26] G.N. GaticA, An augmented mixed finite element method for linear elasticity with nonhomogeneous Dirichlet conditions, Electron. Trans. Numer. Anal.. 26 (2007), pp. 421-438.

[27] G.N. Gatica, A Simple Introduction to the Mixed Finite Element Method: Theory and Applications, Springer Briefs Math., Springer, Cham, Switzerland, 2014.

[28] G.N. Gatica, M. González, and S. Meddahi, A low-order mixed finite element method for a class of quasi-Newtonian Stokes flows. I. A priori error analysis, Comput. Methods Appl. Mech. Engrg., 193 (2004), pp. 881-892.

[29] G.N. Gatica And G.C. Hsiao, On the coupled BEM and FEM for a nonlinear exterior Dirichlet problem in $\mathrm{R}^{2}$, Numer. Math., 61 (1992), pp. 171-214.

[30] G.N. Gatica, A. Márquez, and S. Meddahi, An augmented mixed finite element method for $3 D$ linear elasticity problems, J. Comput. Appl. Math., 231 (2009), pp. 526-540.

[31] G.N. Gatica, A. Márquez, R. Oyarzúa, and R. Rebolledo, Analysis of an augmented fullymixed approach for the coupling of quasi-Newtonian fluids and porous media, Comput. Methods Appl. Mech. Engrg., 270 (2014), pp. 76-112.

[32] G.N. Gatica, A. Márquez, And M.A. SÁnchez, Analysis of a velocity-pressure-pseudostress formulation for the stationary Stokes equations, Comput. Methods Appl. Mech. Engrg., 199 (2010), pp. 1064-1079.

[33] G.N. Gatica, A. Márquez, and M.A. SÁnchez, A priori and a posteriori error analyses of a velocity-pseudostress formulation for a class of quasi-Newtonian Stokes flows, Comput. Methods Appl. Mech. Engrg., 200 (2011), pp. 1619-1636.

Copyright $@$ by SIAM. Unauthorized reproduction of this article is prohibited. 
[34] G.N. Gatica, And W.L. Wendland, Coupling of mixed finite elements and boundary elements for linear and nonlinear elliptic problems, Appl. Anal., 63 (1996), pp. 39-75.

[35] J.S. Howell, Dual-mixed finite element approximation of Stokes and nonlinear Stokes problems using trace-free velocity gradients, J. Comput. Appl. Math., 231 (2009), pp. 780-792.

[36] J.S. Howell and and N.J. Walkington, Inf-sup conditions for twofold saddle point problems, Numer. Math., 118 (2011), pp. 663-693.

[37] J.S. Howell and N.J. Walkington, Dual-mixed finite element methods for the Navier-Stokes equations, ESAIM Math. Model. Numer. Anal., 47 (2013), pp. 789-805.

[38] J. NEČAS, Introduction to the Theory of Nonlinear Elliptic Equations, John Wiley \& Sons, Chichester, England, 1986.

[39] R.Oyarzúa, T. QIn, AND D. Schötzau, An exactly divergence-free finite element method for a generalized Boussinesq problem, IMA J. Numer. Anal., 34 (2014), pp. 1104-1135.

[40] A. Quarteroni And A. Valli, Numerical Approximation of Partial Differential Equations, Springer Ser. Comput. Math. 23, Springer-Verlag, Berlin, 1994.

Copyright (C) by SIAM. Unauthorized reproduction of this article is prohibited. 